\title{
Nuclear Polymer Explains the Stability, Instability, and Nonexistence of Nuclides
}

\author{
Dirk J. Pons, ${ }^{1}$ Arion D. Pons, ${ }^{2}$ and Aiden J. Pons ${ }^{3}$ \\ ${ }^{1}$ Department of Mechanical Engineering, University of Canterbury, Private Bag 4800, Christchurch 8020, New Zealand \\ ${ }^{2}$ University of Canterbury, Christchurch 8020, New Zealand \\ ${ }^{3}$ Rangiora New Life School, Rangiora 7400, New Zealand \\ Correspondence should be addressed to Dirk J. Pons; dirk.pons@canterbury.ac.nz
}

Received 12 September 2014; Revised 16 April 2015; Accepted 30 April 2015

Academic Editor: Ali Hussain Reshak

Copyright (C) 2015 Dirk J. Pons et al. This is an open access article distributed under the Creative Commons Attribution License, which permits unrestricted use, distribution, and reproduction in any medium, provided the original work is properly cited.

Problem. The explanation of nuclear properties from the strong force upwards has been elusive. Approach. Design methods were used to develop conceptual mechanics for the bonding arrangements between nucleons, based on the covert structures for the proton and neutron as defined by the Cordus theory, a type of nonlocal hidden-variable design with discrete fields. Findings. Nuclear bonding arises from the synchronous interaction between the discrete fields of the proton and neutron. This results in not one but multiple types of bond, cis- and transphasic, and assembly of chains and bridges of nucleons into a nuclear polymer. The synchronous interaction constrains the relative orientation of nucleons, and hence the nuclear polymer takes only certain spatial layouts. The stability of nuclides is entirely predicted by morphology of the nuclear polymer and the cis-/transphasic nature of the bonds. The theory successfully explains the qualitative stability characteristics of all hydrogen and helium nuclides. Originality. Novel contributions include the concept of a nuclear polymer and its mechanics; an explanation of the stability, instability, or nonexistence of nuclides starting from the strong/synchronous force; explanation of the role of the neutron. The theory opens a new field of mechanics by which nucleon interactions may be understood.

\section{Introduction}

The internal structural relationships of the atomic nucleus are poorly understood. Although quantum chromodynamics (QCD) offers an explanation of intranucleon bonding in terms of color force, the internucleon bonding via the residual strong force is poorly understood. Neither how binding energy arises nor why the neutrons are necessary at all is clear. Models, such as binding energy, represent the empirical value of nuclide properties but do not explain nuclide stability. All these approaches have their place but are fragmentary and lack integration. None of the theories or models, singly or collectively, is able to explain nuclear properties from the strong interaction upwards. And it has not been possible to explain, from first principles, why any nuclide is stable, unstable, or nonexistent.

This paper contributes to solving this problem. It develops a conceptual framework for a solution on the basis of a proposed new physics at the foundational level. More specifically, the paper takes an existing but unusual conjectured design for the structure of particles at the fundamental level, namely, the nonlocal hidden-variable (NLHV) design of the Cordus theory, and from that develops a conceptual theory for the arrangement of the atomic nucleus. The theory at the foundational level is based on the geometric internal structures of particles, and consequently the nuclear theory that emerges is also a geometric one, as opposed to a mathematical formalism.

In this context, "hidden-variable" refers to the proposition that particles have internal structure. In contrast, classical mechanics and relativity are based on continuum mechanics, and quantum mechanics (QM) is based on the presumption of zero-dimensional (0D) points. In QM, particles have properties such as charge, spin (orientation), and mass, but these are deemed intrinsic variables of a mathematical rather than physical origin. Hidden-variable theories assert that particles have internal structures that give rise to the externally evident properties. Such theories 
are therefore premised in physical realism: if an observable property exists, then there must be an underlying physical mechanism that causes it [1]. The hidden-variable approach is therefore consistent with the principles underlying the scientific method. However, it is an obscure approach because of the historical difficulty in finding such solutions, a difficulty which has only recently been overcome.

Hidden-variable designs come in two types: local and nonlocal. The local theories assume that the principle of locality applies. Locality means that the behavior of a particle is only affected by fields, forces, and phenomena at the point at which the particle exists, not by the values of fields or effects elsewhere. Related to this is local realism whereby the properties of an object preexist before the object is observed. The Bell type inequalities [2-4] preclude local hidden-variable solutions, which mean that $0 \mathrm{D}$ point particles are theoretically incapable of having internal structure. However this is unsurprising given that locality is closely aligned to QM's premise that particles are $0 \mathrm{D}$ points. The many experiments with entanglement are generally interpreted as supporting superluminal causality and nonlocal behavior; that is, locality does not to apply at the foundational level. This introduces a major incongruity in that physics at the macroscopic level of continuum mechanics is otherwise believed to be local. The nonlocal hidden-variable theories, for example $[5,6]$ and more recently [7], accept that entanglement effects are real. They propose mechanisms based on physical causation to explain the phenomena.

There are no grounds, neither of physical realism nor of mathematical disproof, that disallow NLHV theories as a class. This is not contentious. However, nonlocal theories have their own difficulties in that they have been hard to discover, with only a few viable candidates. The de BroglieBohm pilot-wave theory $[5,6]$ is one such solution, but it has not progressed beyond an explanation of the doubleslit behavior of light, cannot explain other phenomena, and cannot function as an independent theory of physics generally. More recent nonlocal developments in the form of the Cordus theory [7] have much greater general applicability, that is, improved external construct validity. The present paper shows how this latter theory may be extended, in a conceptual manner, to provide a novel qualitative explanation of nuclear structure. It shows, using this Cordus nonlocal hidden-variable design, that it is possible to explain nuclear structure from the strong force, which has not previously been achieved for any theory of physics. The theory is the first to explain why any nuclide from $\mathrm{H}$ to $\mathrm{Ne}$ is stable, unstable, or nonexistent. Even the relative trends in lifetime can be qualitatively explained. The role of the neutron in nuclear bonding is elucidated, which also has not previously been achieved. The depth of explanations achieved is greater than that provided by competing theories. This has the profound implication that the next physics may be based on nonlocal hidden-variable designs rather than zero-dimensional point particles.

This paper sets out the underlying assumptions of this theory, describes its mechanics, and applies these to the nuclides of hydrogen and helium. Other works extend this further, to include all the nuclides up to and including neon.

\section{Existing Approaches to Nuclear Structure}

The interaction between nucleons is not known with confidence. Consequently, a direct computation of the Schrödinger equation is not feasible for anything but the simplest atoms [8]. Quantum chromodynamics proposes an explanation of the bonding of quarks inside the nucleons, by the exchange of gluon in the color force $[9,10]$. It is generally believed from consideration of the density of the nucleus that the strong force has a short range, so that nucleons are only attracted to other local nucleons, not by the bulk of nucleons as a whole (which would increase the density beyond that observed). Density considerations also suggest that the force is repulsive at closer ranges, so the nucleons are unable to come too close. However, QCD does not fully explain all the characteristics of the strong force and is unable to explain how the proton and neutron bond. It thus has little to contribute to our understanding of how multiple nucleons might interact.

More comprehensive nuclear models are the liquid drop model [11], semiempirical mass formula (SEMF) [12], and Ivanenko's shell model [13]. The nature of these models is to approximate nuclear characteristics with mathematical representations. They include terms to accommodate the strong and electrostatic forces. However, these theories mathematically model, rather than explain, the binding energy (BE) characteristics. They do this reasonably accurately, which might be considered a success if not for the fact that they are disconnected from any theory of the strong force. Anyway, binding energy only correlates weakly with nuclide stability. A further problem is that the models treat the neutrons and protons independently: there is an assumption that the particles are different and therefore occupy different quantum states. However, it is also apparent from observation that no nucleus exists with multiple protons and no neutrons, so evidently neutrons provide an important role within the nucleus, which is not represented in any of the existing theories. Similar problems apply to the interacting boson model $[14,15]$ which assumes that nucleons exist in pairs. However, this limits the model to nuclides where $\mathrm{p}=\mathrm{n}$, which is an overly simplistic assumption.

Another problem that none of the models overcomes is how the nucleus is held together. The liquid drop and SEMF treat the nucleons as point particles uniformly distributed in a volume. The models require there some bonding between nucleons but do not identify the mechanism. Furthermore, the repulsive nature of the strong force at short range is excluded from the nuclear models. A related problem is how the volume of the nucleus arises. The models can provide a mathematical fit to the empirical data for charge radii [16]; yet none of the models explains how the aggregation of $0 \mathrm{D}$ point particles creates geometric size. Deeply problematic is the disconnect between the QCD strong force and the nuclear models, as already mentioned.

These theories attempt to solve the quantitative part of the problem, because that is amenable to the mathematical modeling method, but this is not the real problem. The real need is to differentiate stable from unstable nuclides, but none of these theories is able to do this. Logically consistent physics should be able to explain how the strong force 
causes nuclear structure, but this has not been achieved. No existing theory, or collection of theories, can explain the mechanisms whereby the strong interaction causes nuclear structures. A core unresolved problem in nuclear theory is how protons and neutrons interact. Inspection of the empirical evidence in the table of nuclides shows that the assumption of independence of the nucleon particles cannot be valid. The stability is not determined simply by quantity of nucleons, as if protons and neutrons contributed equally. The magic number approach does not generalize to explain the table of nuclides as a whole. Instead, stability of a nuclide is an unknown function of the number of both protons and neutrons. Also the evidence clearly shows that neutrons play an essential role in stability, though the trends are complex and the underlying mechanics are unknown. There is a need to find better theories describing how protons and neutrons interact, before the nuclides can be understood.

\section{Purpose and Approach}

The purpose of this paper was to develop a systematic theory to explain the relationships between nucleons and how this results in the stability characteristics of the nuclides. The particular objective was to explain the stability, instability, and nonexistence states of the nuclides.

The approach started with a specific design for matter. This was the covert structure defined by the Cordus theory [7], which is a combination of a nonlocal hiddenvariable design with externally propagating discrete fields. The hidden-variable approach was selected as it has shown excellent ability to solve many other complex problems in physics [17-20]. Seeking solutions in the hidden-variable sector is an unusual approach, as mainstream physics is generally dismissive of this sector. The usual objection is that local hidden-variable solutions are precluded by the Bell type inequalities [2-4]. Nonetheless, it is relevant to note that the inequalities do not preclude all nonlocal hiddenvariable designs $[21,22]$. A more serious limitation has been the inability of this sector to yield solutions, other than the de Broglie-Bohm pilot-wave theory $[5,6]$ which has limited applicability. That limitation has been overcome by the advent of the Cordus theory, which is a type of NLHV design. A second reason for selecting the hidden-variable sector is that the theories based on a $0 \mathrm{D}$ point premise have been unable to explain the nuclides, and therefore it is worth attempting other approaches. The results justify this decision by showing that it is indeed possible to explain the nuclides this way.

The method was conceptual, that is, a Gedankenexperiment, using the design method. This was applied to infer the structural arrangements between the nucleons that would be sufficient to provide the observed behavior of the nuclides. The design was also required to maintain logical internal consistency, that is, not to contravene the other parts of the theory. Necessary assumptions were recorded as lemmas. This design method is described more fully elsewhere [23, 24 . The result is a conceptual theory, expressed qualitatively.

The Cordus theory is built on the proposition that all particles have internal structures and emit discrete forces. More specifically, particles are proposed to comprise two reactive ends some distance apart, with the reactive ends energized in turn at a frequency (the de Broglie frequency), at which time they emit discrete forces [25]. These discrete forces propagate into the external environment and are connected in flux lines (Cordus: hyperfine fibril or hyff) [7]. They make up the fields. The two reactive ends are joined by a fibril (hence Cordus) that does not interact with matter, and which instantaneously coordinates the phase of the two ends. Thus, there is an inner structure and an external system of discrete forces. This structure is called a particule. Within this theoretical framework, the strong force is mediated by the synchronicity of discrete forces emitted by NLHV particules [17]. Thus, the strong force is reconceptualized as a synchronous interaction. More details about the particule idea are available elsewhere, including development of the theory to explain wave-particle duality [7], unification of the electromagnetic-gravitational and strong forces [17], explanations for antimatter and the process of annihilation [18, 24], and a theory for time [19]. The present paper applies the design method to identify how such a synchronous interaction might operate on nucleons. The result is a conceptual theory that predicts a specific type of geometric layout of nucleons in the nucleus and is called a nuclear polymer. The mechanics of this polymer are identified and noted as lemmas in Appendix A of the supplementary material; see Supplementary Material available online at http://dx.doi.org/10.1155/2015/651361.

\section{Results}

First, the predicted structures of the proton and neutron are described. A logical consequence of the theory is that such structures will form bonds with the synchronous interaction (strong force). Surprisingly, multiple types of bonds are predicted, which are termed cisphasic and transphasic for reasons which will become obvious. This is a radical departure from all $0 \mathrm{D}$ point based theories and models. It leads to a conceptual breakthrough in the form of a predicted spatial arrangement of the nucleons. These principles are then used to determine the designs for the hydrogen and helium nuclides, and these are shown to have excellent fit to the two isotope series.

4.1. Proton and Neutron Structures. The Cordus theory for the proton is shown in Figure 1. The derivation of this structure is shown in the references and is not repeated here.

The HED notation is a Cordus symbolic representation of the distribution of the discrete forces in the three-emission directions and is a unique signature for the type of particule [24]. As described in [17], the proton has two discrete forces in one of the axes and hence the designation $\mathrm{p}\left(r_{1.1}^{1} \cdot a_{1} \cdot t_{1}\right)$.

The Cordus theory for the neutron is shown in Figure 2. This theory also explains why the neutron is unstable when alone. This is because, while it has a unit charge (neutral), its HEDs are incompletely energized. Specifically the $[t]$ axis is unenergized and is vulnerable to disturbance by discrete forces of external origin.

These predicted covert structures for the proton and neutron are plausible and are not precluded by the Bell-type 
Proton $\mathrm{p}$
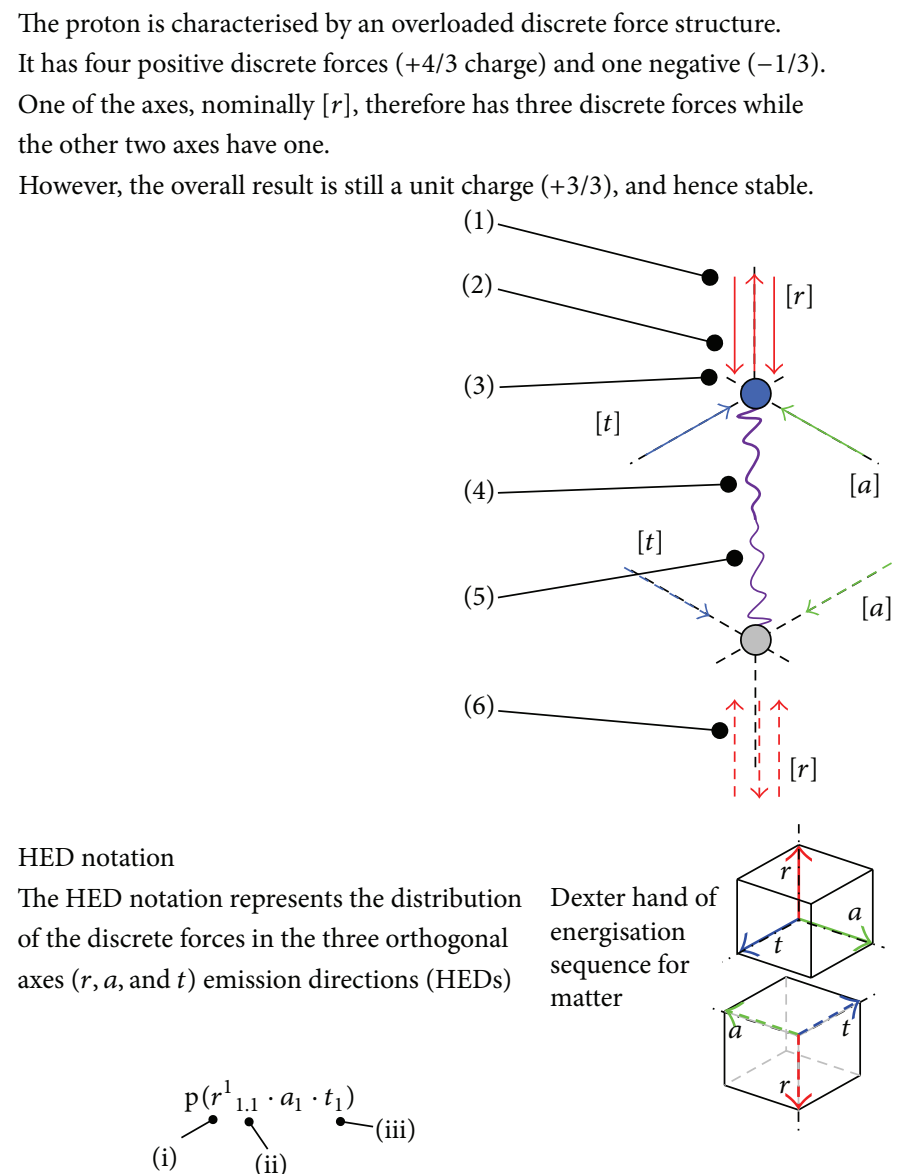

FIGURE 1: The predicted NLHV and discrete field structures of the proton. One of these axes has an extra pair of discrete forces, giving four discrete forces in total but still a net charge of +1 . (1) HEDs accept multiple discrete forces. (2) Proton has two extra discrete forces, but these balance each other. (3) Hand is identical to that of electron, but emission direction is reserved (charge). (4) Type of reactive end: oscillating. One reactive end energizing and the other deenergizing one ( $180^{\circ}$ out-of-phase). (5) Span is inversely proportional to frequency (and mass). So proton span is much smaller than electron's. (6) Some flexibility about location of the extra discrete forces may be possible. (i) Axis is overloaded with discrete forces. (ii) Two discrete forces. (iii) Each discrete force carries a $+1 / 3$ electrical charge and so overall +1 charge.

inequalities or any other theory. The proton and neutron structures may look exotic, but that is only because the dominant way of thinking about particles is as $0 \mathrm{D}$ points. They are not inconsistent with other theories of physics, that also have their intrinsic variables or hidden dimensions. A coarser level representation is obtained when the span is reduced to zero, and this corresponds to the $0 \mathrm{D}$ point approximation of QM. The number of parameters needed to describe a Cordus particule is consistent with the number of dimensions in some string/M theories.

Note that in this theory each particule has two ends, and there is a span, that is, a physical distance between the two reactive ends. This means that particules and their assemblies (described below) have physical size; there is no singularity as with QM. Also the particule energizes its reactive ends sequentially, at its frequency, and the reactive ends are not both simultaneously in the same state. Hence a phase exists, with further implications for bonding (described below). The concept of particules having physical size (span), phase, and spatially orientated discrete emissions is core to this theory and permits a revolution in the understanding of the nucleus.

4.2. Phased Behavior between Particules. The Cordus theory proposes that the strong force arises from the synchronization of discrete forces between the reactive ends of different particules [17]. The emission directions represent the particule's directional engagement with the external environment, and so two particules that colocate one of each of their reactive ends need to share this access, and this is proposed as the basis for the synchronicity requirement. This causes the emission of the particules' discrete forces to be interlocked. The discrete forces cause the reactive ends to be pulled into (or repelled from) colocation and held there and hence the strong nature of the forces, its apparent attractive-repulsive nature, and its short range. Unexpectedly, the Cordus theory predicts that this synchronous force only applies to particules in coherent assembly. In such situations, the synchronicity of emission means also that the assembled particules must energize at the 


\section{Neutron $n$}

The neutron is characterised by having two discrete fields in each direction (charge) and is therefore neutral as a whole.

However, it does not have full HEDs in all three axes and is therefore intrinsically instable, unless free to dynamically reallocate the discrete fields, or is bonded with a proton.

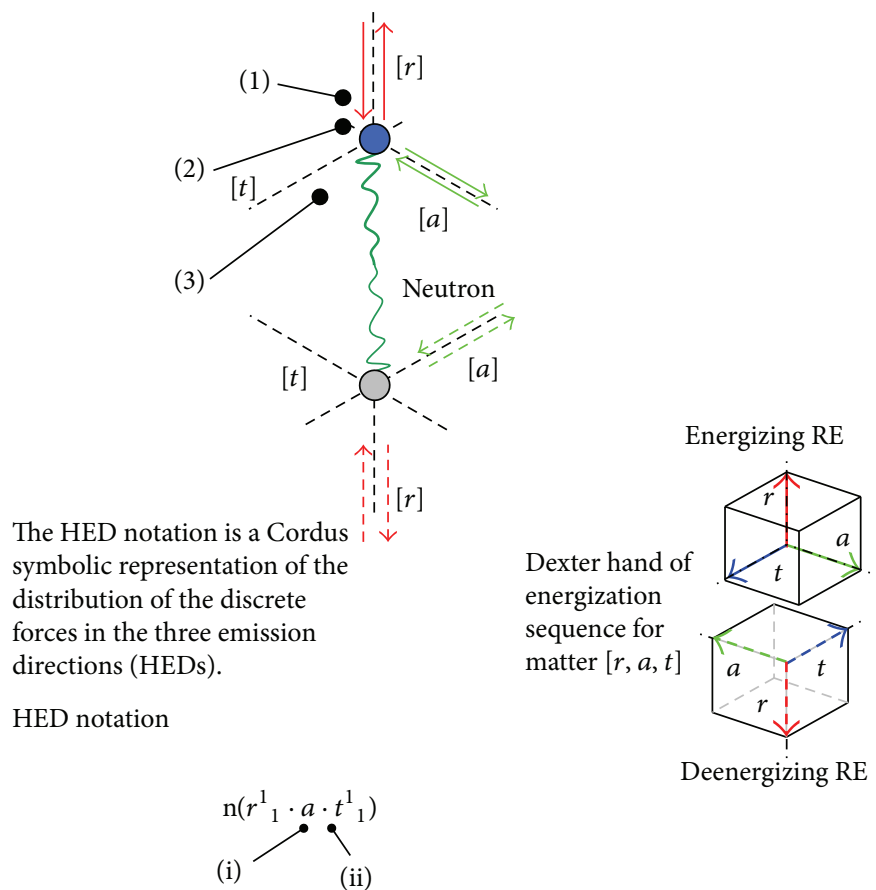

FIgURE 2: Predicted structure of the neutron. The HED arrangements provide a neutral pair of discrete forces, comprising a positive and negative force, on two of the emission directions. The overall charge is thus neutral. It is believed that the pairs may shift to other axes as necessary. (1) Two opposed discrete fields in the HED and hence neutral charge. (2) Only two HEDs contain discrete fields pairs. (3) Anticipate that discrete fields may dynamically relocate to other HEDs when required by bonding. (i) Arrangement of discrete fields is flexible in neutron: pairs may shift to $[a]$ axis. (ii) Each discrete field carries a 1/3 signed electrical charge and so overall neutral charge.

same frequency (or a suitable harmonic), either in- or outof-phase. Thus, the synchronous interaction is predicted to be limited to particules in coherent assembly relationships, with the electromagnetic-gravitational forces being the corresponding interaction for discoherent assemblies of matter.

The next step in the creation of a nuclear model is to find a candidate set of principles for the assembly of protons and neutrons using this synchronous interaction. Consider two particules that are in a coherent assembly, that is, have a common magnitude of frequency, and share a common location for at least one reactive end each. There are two states that particularly interest us: the particules may be in-or outof-phase with each other at their common location, and these are termed these cis-and transphasic behavior, respectively [17]. The principles are believed to apply to all particules, including the electron, but the focus here is on the assembly relationships between a proton $(\mathrm{p})$ and neutron $(\mathrm{n})$.

4.3. Cisphasic Interactions. Cisphasic bonding (denoted by \#) occurs where reactive ends from two or more particules are colocated and are in-phase. The particules must have complementary discrete forces emissions; that is, there must be some advantage gain in the combined discrete forces. Typical advantages might be summation to a whole unit of charge (e.g., the UUD quark complement of the proton) or a more spatially uniform set of emissions (e.g., the protonneutron bond will be shown).

By complementary is meant that the one particule provides discrete forces that the other lacks, and this is reciprocated. This generally means they must be different types. The case in point is the bonding of a proton and neutron: their discrete forces arrangements are very different, but the assembly gives a neatly balanced arrangement with a unit charge and the same number of discrete forces in each of the three directions. This complementary state is best seen by considering the process by which a proton and neutron would come together to form a bond; see Figure 3. The basic idea is that two approaching $\mathrm{p}$ and $\mathrm{n}$ particules, which are not too dissimilar in frequency and phase, negotiate location and phase via their discrete forces and adjust their emissions to move into synchronicity. The synchronous emissions then lock the two reactive ends together.

The cisphasic behavior results in the merging of the reactive ends into a new assembly characterised by high bonding forces and the subcomponents losing much of their 


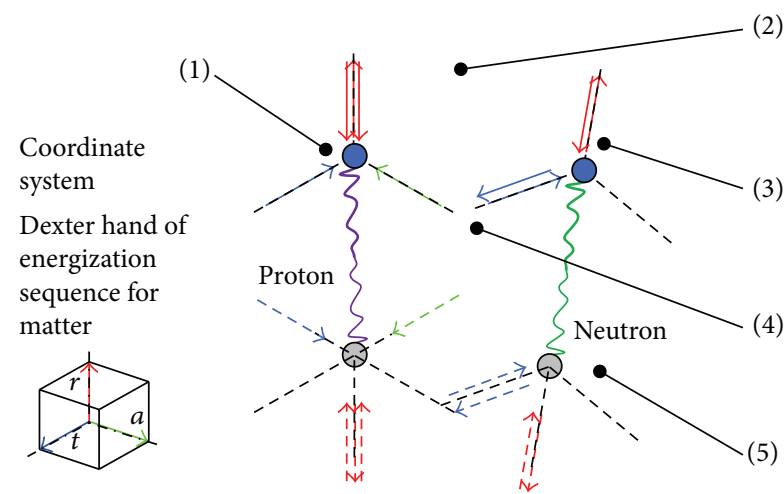

(a) Approximation

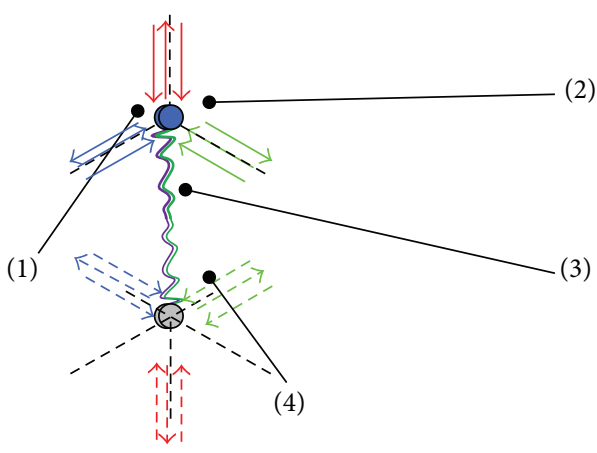

(c1) Parallel assembly (closed)

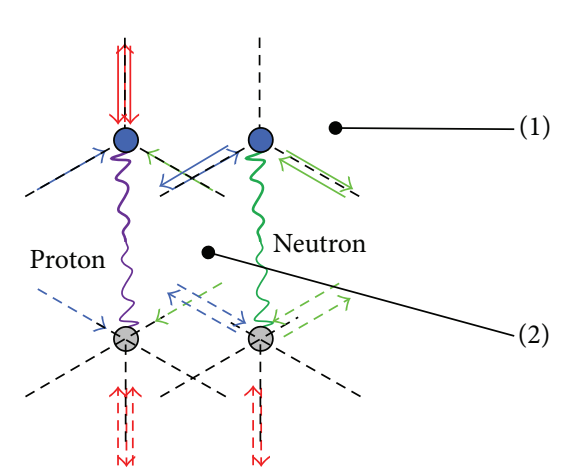

(b) Accommodation

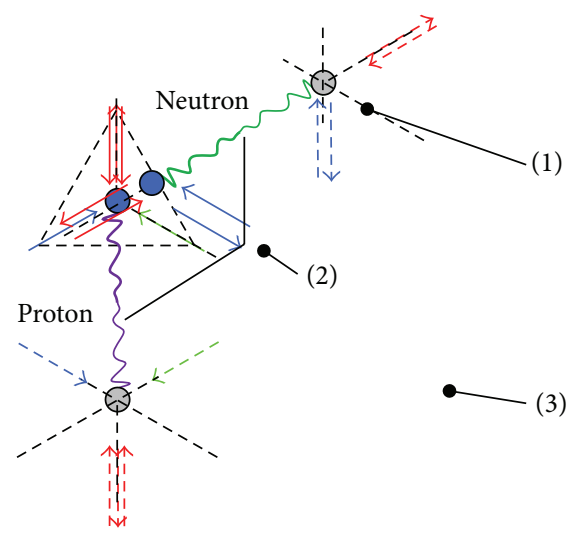

(c2) Series assembly (open)

Figure 3: Cisphasic assembly of proton and neutron ( $\mathrm{p} \# \mathrm{n}$ ), showing the processes and how the two particules complement each other's discrete force emissions. In Figure (a) we have the following. (1) Proton. This is the energizing side. (2) We present this interaction as the neutron doing all the adjustment, though both are involved. (3) Neutron needs to be in a suitable state regarding orientation, frequency, and phase. (4) Particules respond to each other's discrete forces as they come closer. (5) If the state is unsuitable, then the neutron may be repulsed. In Figure (b) we have the following. (1) Neutron rearranges active HEDS to match (both particules may do this). (2) The frequencies of the two particules are pulled into synchronicity. The original individual frequencies would be slightly different, due to the different type of particule (rest mass) and energy. In Figure (c1) we have the following. (1) The particules merge to form a new assembly structure. (2) Each HED now has 2 positive discrete forces (inwards) and one negative (outwards), that is, xll.1 configuration. The total charge is still +1 . (3) The subcomponents lose their individuality and become a new assembly, in this case a pn. (4) This is a closed assembly. In Figure (c2) we have the following. (1) The neutron is able to rearrange its active HEDs to align with the new planes presented by the proton. (2) This is a right angle. (3) For this structure to be stable these open ends, they will need to be joined together, if necessary by other neutrons and protons. The quality of these other joints determines the overall stability of the assembly.

individual identity. The outcome is a new assembly, in this case a pn deuteron, for which the Cordus HED notation is

$$
\begin{gathered}
\mathrm{n}\left(r \cdot a_{1}{ }^{1} \cdot t_{1}{ }^{1}\right)+\mathrm{p}\left(r_{1.1}{ }^{1} \cdot a_{1} \cdot t_{1}\right) \\
\longrightarrow \mathrm{O}\left(r_{1.1}{ }^{1} \cdot a_{1.1}{ }^{1} \cdot t_{1.1}{ }^{1}\right),
\end{gathered}
$$

where $\mathrm{O}$ is the deuteron.

The proposed mechanics are set out in the supplementary material. Note that the output HEDs are balanced regarding discrete forces: the assembled reactive end has two inward discrete forces and one outward, in each of the three emission directions $[r, a, t]$. Consequently this meets plausible expectations of stability. Note also that the output state $\mathrm{O}$ is more balanced, hence better for stability, than either of the input $\mathrm{n}$ or $\mathrm{p}$. This explains why there is advantage in the positive proton bonding with the neutral neutron. The cisphasic proton-to-neutron bond, with its balanced discrete forces and in-phase synchronicity, has more stability advantage and is therefore preferred by the nuclear polymer over the transphasic proton-to-proton bond (described below). It is important to note that all such bonds are not made on the basis of charge, that is, the bond is not electrostatic. Rather the participants are motivated to obtain a more balanced emission of discrete forces, a balance less susceptible to external interference and hence also more stable against decay. This also explains why the neutron is stable when bonded with a proton but unstable on its own. It has incomplete and imbalanced discrete forces which are complemented exactly by the proton, whereas on its own these make it vulnerable to perturbation from random discrete forces in the external environment and hence decay $[20,25]$. 
TABLE 1: Viability of the different proton and neutron combinations.

\begin{tabular}{ll}
\hline $\begin{array}{l}\text { Cisphasic \# (reactive } \\
\text { ends in phase) }\end{array}$ & $\begin{array}{l}\text { Transphasic } \times \text { (reactive ends } \\
\text { out of phase })\end{array}$ \\
\hline Two protons & Two protons \\
$\mathbf{p} \# \mathbf{p}$ & $\mathrm{p} \times \mathrm{p}$ \\
Notviable & Viable \\
\hline Two neutrons & Two neutrons \\
$\mathbf{n} \# \mathbf{n}$ & $\mathrm{n} \times \mathrm{n}$ \\
Notviable & Viable \\
\hline Proton joined to neutron & Proton joined to neutron \\
$\mathrm{p} \# \mathrm{n}$ & $\mathbf{p} \times \mathbf{n}$ \\
Viable & Notviable \\
\hline
\end{tabular}

There are two subtypes of this interaction: parallel and series. In parallel assemblies, each particule joins to the other at both reactive ends. A series assembly is also permitted, where there is only a single partnership between any one proton and neutron. This leads to open chains, which may be closed by other nucleons. The mechanics require that a stable chain may not be terminated by a naked neutron. The openassembly pn deuteron therefore joins with others like it, to form a nuclear polymer.

4.4. Transphasic Interactions. The transphasic joint (denoted by " $\times$ ") also involves colocation of the reactive ends of two particules and synchronous frequency, but the difference is that the reactive ends are at opposite phases and hence the name [23]. Thus the reactive end of one particule energizes at the location while the other deenergizes. This behavior means that the location in question has greater temporal occupancy of discrete forces. It is believed that this protects the particules against interference from external discrete forces (from other particules) and hence promotes stability. The Cordus theory requires that the particules have identical discrete force structures. In contrast, the cisphasic case required complementary structure. This means that the transphasic interaction is only between like particules, for example, $\mathrm{p} \times \mathrm{p}$ or $\mathrm{n} \times \mathrm{n}$ but not $\mathrm{p}$ $\times \mathrm{n}$. The basic structure of a transphasic interaction is shown in Figure 4. As before, both series and parallel arrangements are anticipated.

The Cordus theory for transphasic joints permits neutrons to be joined in extended closed chains. The same transphasic relationship applies to pairs of electrons, thereby explaining electron-pairing, the Pauli exclusion principle, and Cooper pairs. A series transphasic relationship of a skin of electrons explains superconductors.

4.5. Joint Types Available to Protons and Neutrons. The above principles are proposed to be applicable to all particules, including electrons and pions. Applying these principles to the specific case of the nucleons shows that certain joints are allowed and others are not; see Table 1.

Thus, cisphasic bonds only apply to proton-to-neutron joints and the transphasic to bonds between like particules. Thus, it is proposed that the synchronous interaction, when applied to the nucleons, permits the joint types shown in Table 2.
TABLE 2: Summary of the cis- and transphasic joint types and their application to parallel and series assembly structures.

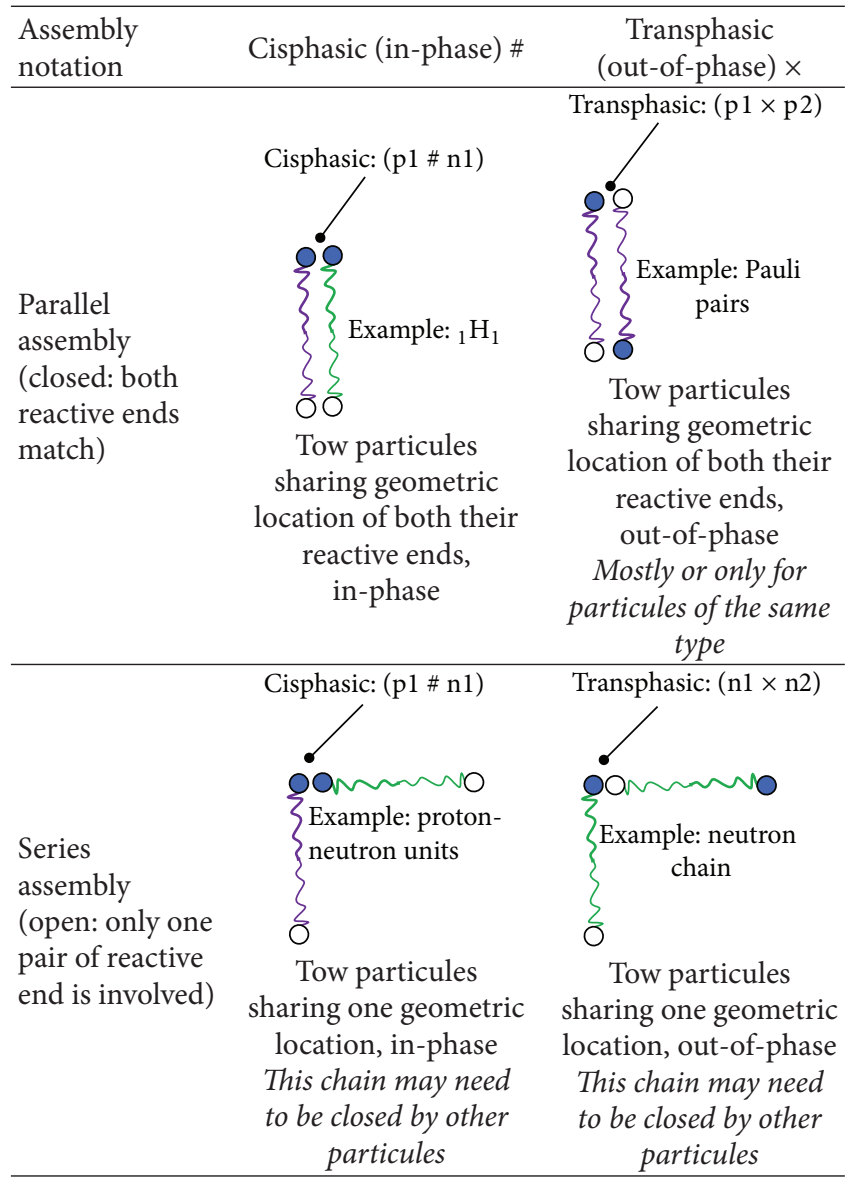

Importantly, this shows that multiple different types of bonds may exist between nucleons. In contrast, all extant nuclear theories and models assume only one type of strong force and assume that the strong and electrostatic forces apply at the same time. The Cordus theory makes a major departure by proposing multiple types of bonds under the synchronous interaction. A logical consequence is that nucleons form into elaborate assembly chains in the form of a nuclear polymer. This too is a novel concept and entirely inaccessible from the standard Model. Next it is necessary to determine, by logical extension of the Gedankenexperiment, what rules apply to this polymer and whether these can explain the nuclides.

4.6. Tendency towards Cubic Structures for the Nuclear Polymer. A logical consequence of the three-dimensional layout of the discrete forces and the handedness thereof [17] is that protons and neutrons preferentially join at right angles and hence cubic structures. The reasons for this are explained in Figure 5.

At each joint, the proton and neutron complement each other's emission of discrete forces; that is there is a synchronous interaction. Specifically, there are three discrete forces in each direction at these junctions, which gives a balanced loading across the three emission directions. 


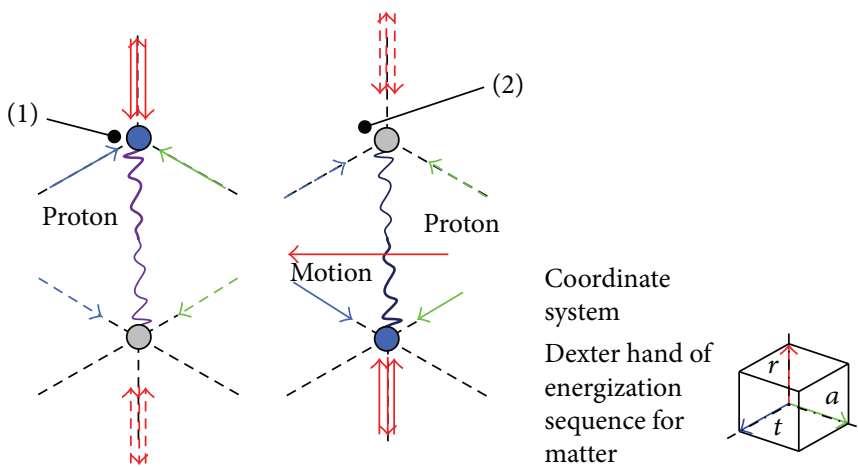

(a) Approximation

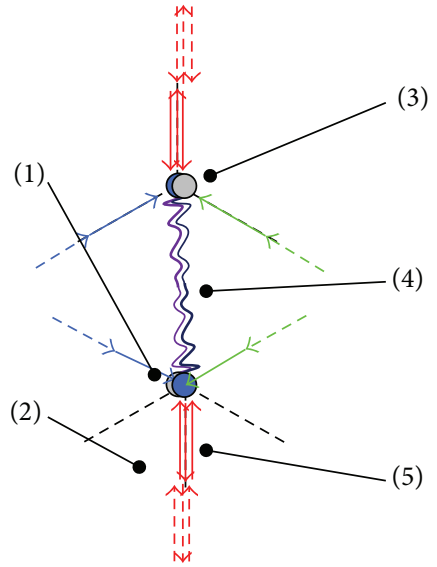

(b1) Parallel assembly (closed)
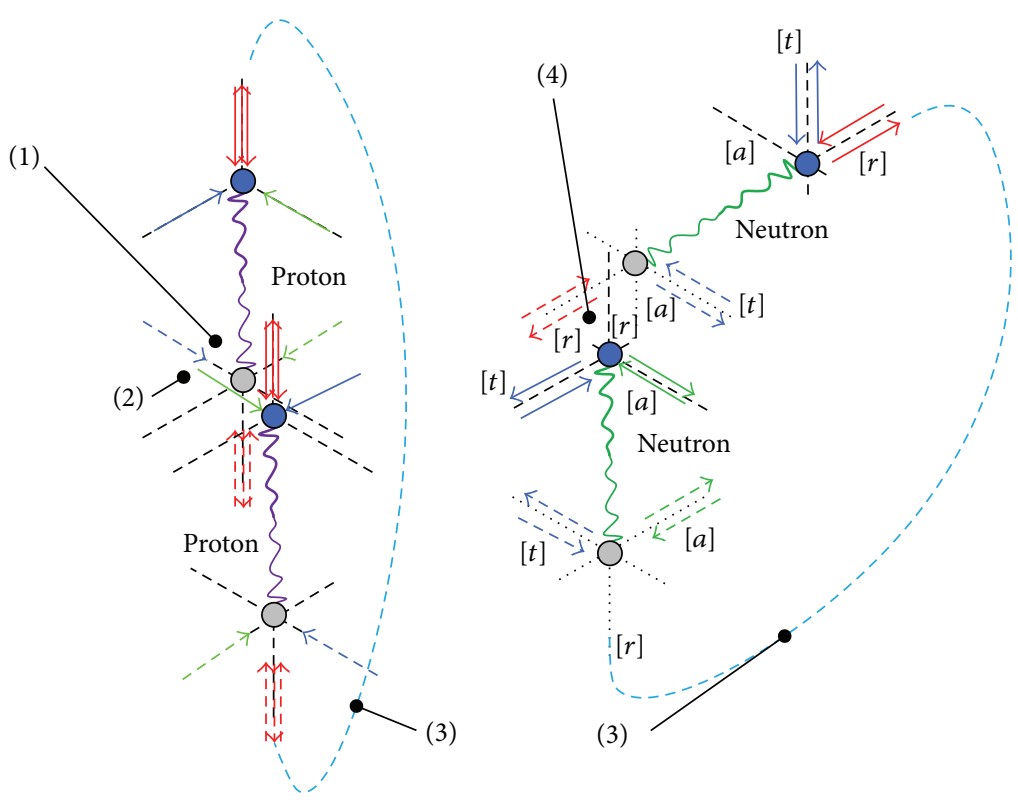

(b2) Series assembly (open)

FIgURE 4: Transphasic joint $(\mathrm{p} \times \mathrm{p})$ : the joining of two particules, two protons in this case, with opposite phase allows them to share the same space. Although illustrated with two protons, this type of bond is available to any pair of like particules, including neutron-neutron and electron-electron. Parallel and series arrangements are available. In Figure (a) we have the following. (1) Proton 1, energizing at this location. (2) Proton 2 is deenergizing, out-of-phase. In Figure (b1) we have the following. (1) The structure is shown closed, but open structures (closed by other chains) are permissible. (2) The need to preserve the same reenergizing locations means that the energy systems of participating particules are coupled together. They can redistribute incoming energy between them and hence also entanglement. (3) Reactive ends from the two protons share the same location, but only one is energizing at any one time. (4) The interaction provides a level of bonding, because the reenergizing locales are protected from outside interference. (5) However, the relationship is one of mutual association, and the particules retain their individual identity. In Figure (b2) we have the following. (1) Two protons with a transphasic assembly. (2) The three axes (HEDs) are not filled equally, so this is unstable. (3) For these structures to be stable, the open ends need to be joined together, by other neutrons or protons. (4) Two neutrons with a transphasic assembly.

Due to the orthogonality of the discrete forces underlying the synchronous bonds, the assembly of multiple particules intrinsically follows a cubic structure. Thus, the nuclear polymer follows a locus around the edges of a set of connected three-dimensional cubes [23].

4.7. Cross Bridges. The nuclear polymer consists, in the first place, of protons and neutrons in series. However, the theory logically permits bridges to form across the polymer. The theory for these bridges is shown in Figure 6.
The theory allows both proton and neutron bridges but predicts that certain configurations will be nonviable. See the lemmas in Appendix A of the supplementary material for details.

4.8. Nuclear Polymer. To sum up the development so far, the theory proposes that the proton and neutron are linear structures (have length), bond in a variety of ways, and form polymers with linear and bridge components, with the polymer being wrapped along the edges of interconnected 


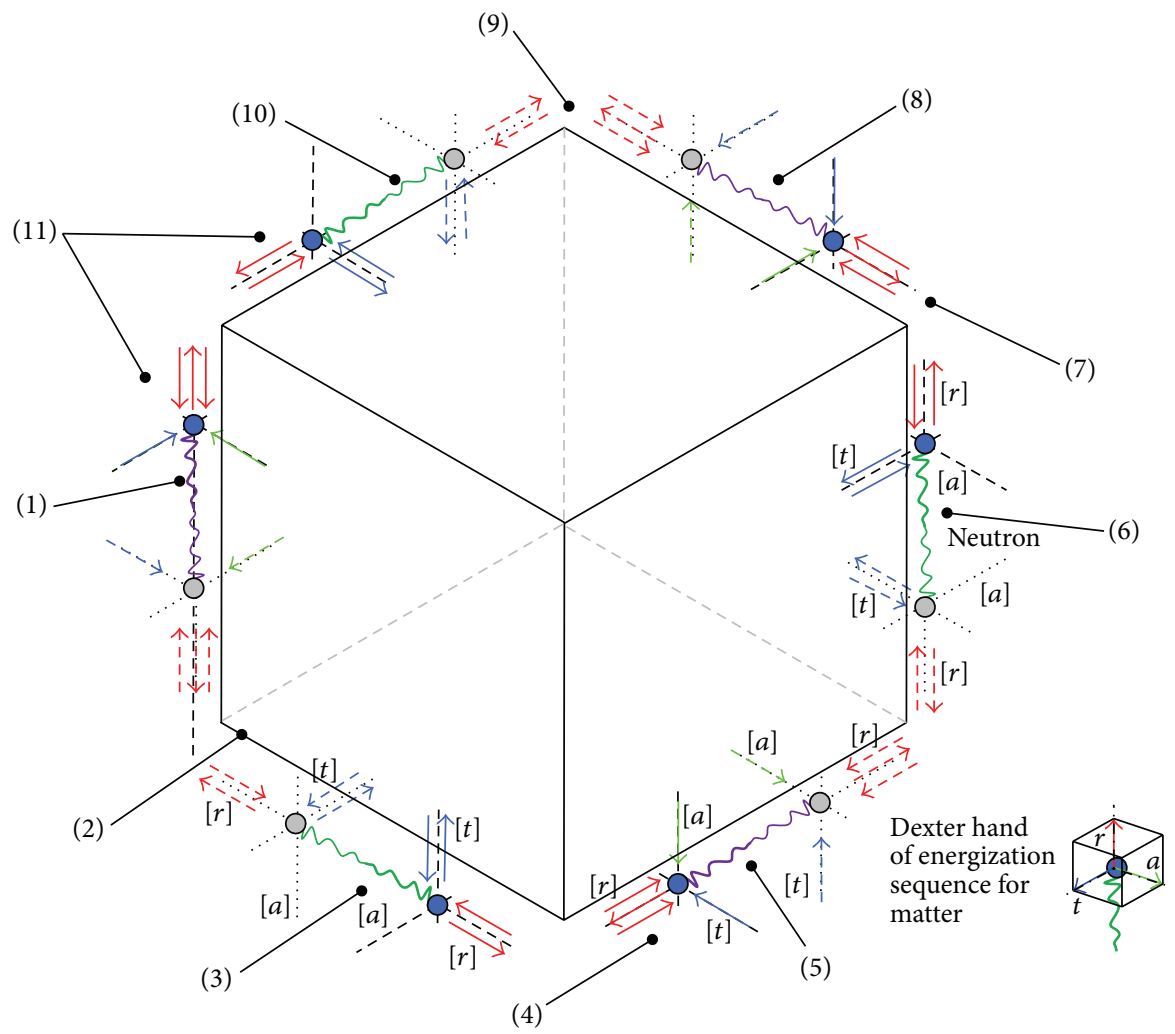

(a) Exploded assembly of three protons (p) and three neutrons (n)

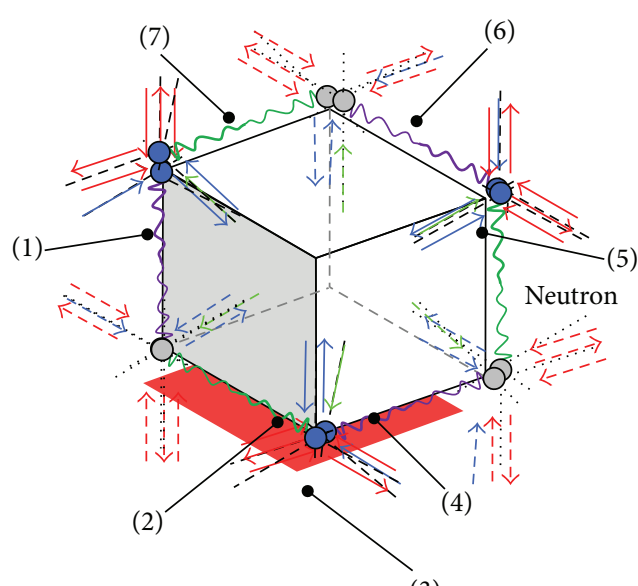

(3)

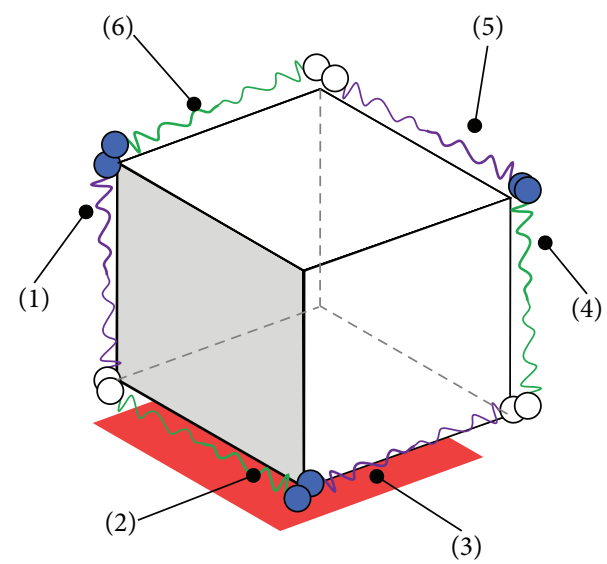

(c) Simplified assembly diagram for the same structure

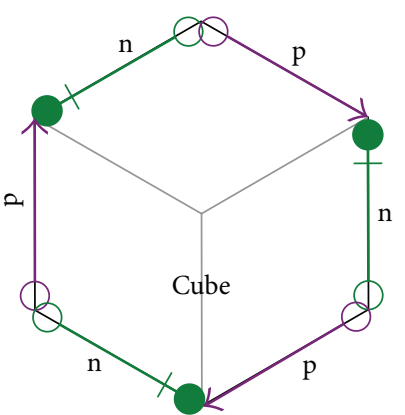

(d) Reduced notation

(b) Assembly of three protons (p) and three neutrons (n) for example, ${ }_{3} \mathrm{Li}_{3}$

FIGURE 5: Cubic structures tend to arise from the bonding of protons and neutrons into a nuclear chain. The diagram shows exploded and assembly views. In Figure (a) we have the following. (1) Proton p1. (2) This figure represents a three-dimensional cube, not a hexagon. (3) n3. (4) Discrete forces shown solid for energizing side and dashed for deenergizing. (5) p3. (6) n2. (7) Note that all the joints in this particular structure are CIS-PHASIC. (8) Proton p2. (9) Discrete forces shown solid for energizing side and dashed for deenergizing. This corresponds to phase. (10) Neutron n1. (11) Note that same phase of these two reactive ends from proton and neutron and hence CIS-PHASIC. In Figure (b) we have the following. (1) p1. (2) n3. (3) Note that at each joint the discrete forces complement each other. There is the same number of discrete forces in each of the three directions. (4) p3. (5) n2. (6) p2. (7) n1. In Figure (c) we have the following. (1) p1. (2) n3. (3) p3. (4) n2. (5) p2. (6) n1. 


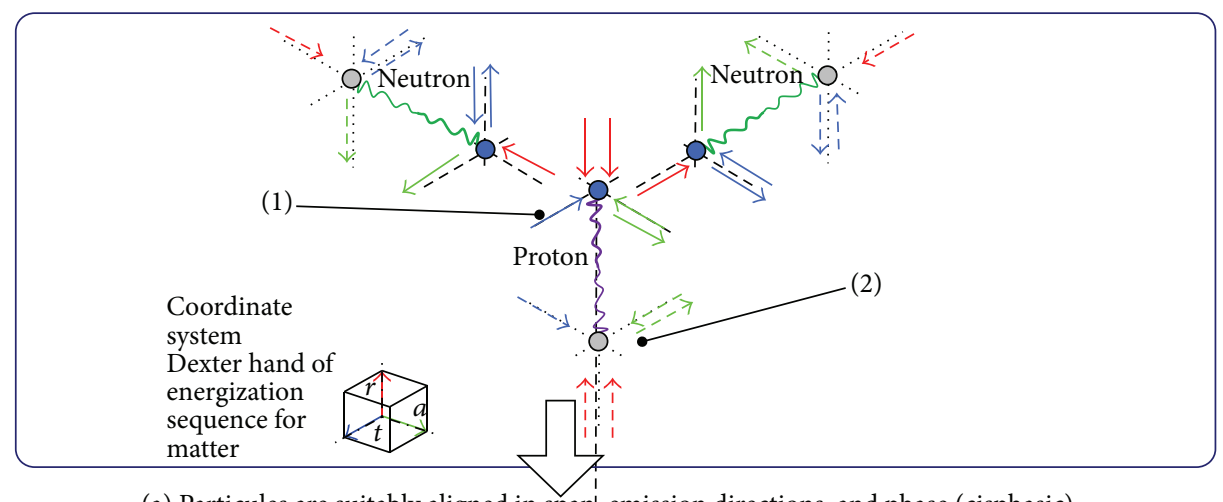

(a) Particules are suitably aligned in span! emission directions, and phase (cisphasic)

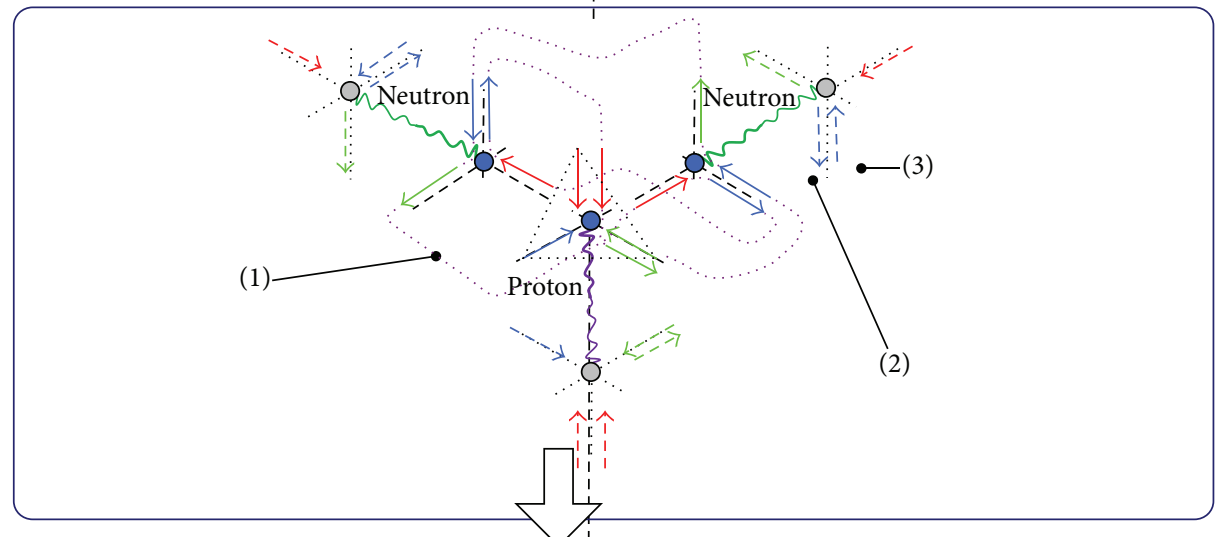

(b) Emission sequences are synchronized

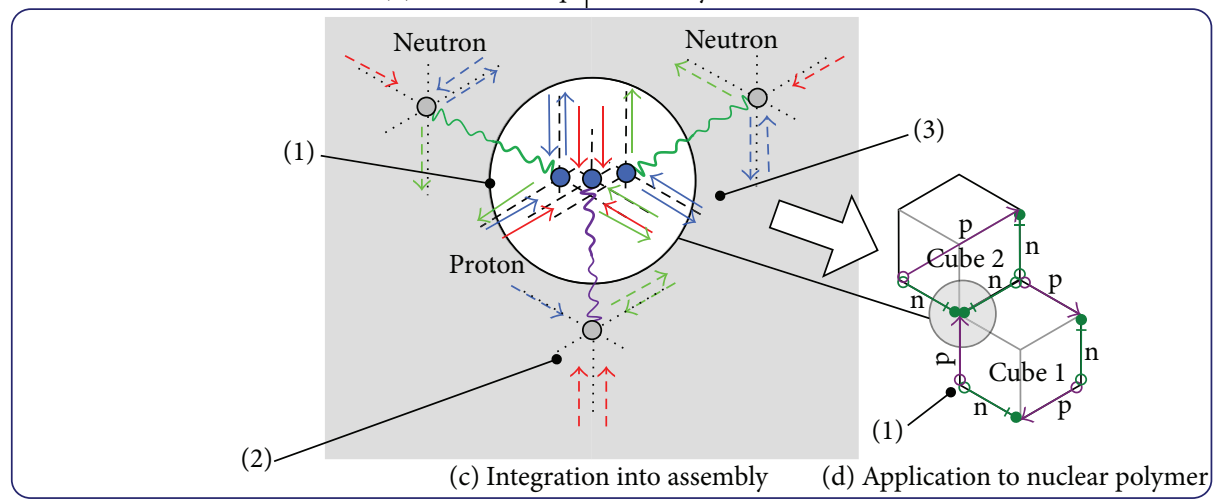

FIGURE 6: Neutron cross-bridges are anticipated to occur within the nuclear polymer. These result in accumulation of discrete forces at the common node. In Figure (a) we have the following. (1) The proton and neutrons each have their own arrangements of discrete forces. We presume these may be configured, something like this. (2) HEDs: three orthogonal hyperfine-fibril emission directions. In Figure (b) we have the following. (1) Particules also have an emission sequence across the HEDs which also creates the matter-antimatter species differentiation. By synchronization, we do not mean that particules emit all their discrete forces at once. Instead assembled particules need to synchronize their emissions into these sequences. One conjectured assembly locus of energization is shown here. (2) By synchronizing emissions of discrete forces (strong force), the particule gains more complete HEDs and hence greater stability. This is particularly important for the neutrons, which otherwise have incomplete HEDs (hence decay when isolated). (3) The assembly locus of energization is also proposed as the mechanism for the generations of matter, but the causality is incompletely understood. In Figure (c) we have the following. (1) The locus of energization pulls the reactive ends together and holds them there while the synchronization remains. This causes one reactive end from each particule to colocate in an assembly. (2) The other reactive end is free to make other arrangements, for example, bonds-even of a different type-with another particule. These other arrangements propagate their effects superluminally to the assembled reactive end. Thus, some perturbation of ANY part of the assembly or its periphery affects the whole. It is therefore important, if stability is to be achieved, that the entire extended assembly is stable. (3) The sum of the discrete forces in HED notation from the proton perspective is $\left(r^{1.1}{ }_{1.1 .1} a^{1.1}{ }_{1.1 .1} t_{1.1}^{1}\right)$ which is still a full unit of positive charge. (For HED notation see http://dx.doi.org/10.5539/apr.v5n5107). In Figure (d) we have the following. (1) The triple-joint enables a bridge neutron configuration within the nuclear polymer. The example shows ${ }_{4} \mathrm{Be}_{5}$. Additional principles determine where the bridge neutrons are permitted. 


$$
{ }_{1} \mathrm{H}_{0} \begin{gathered}
\text { Atomic } \\
\text { hydrogen } \\
\text { (stable) }
\end{gathered}
$$

At the most basic level, the simplest nucleus consists of a single proton with a particule structure. The single proton can exist with its ends exposed.

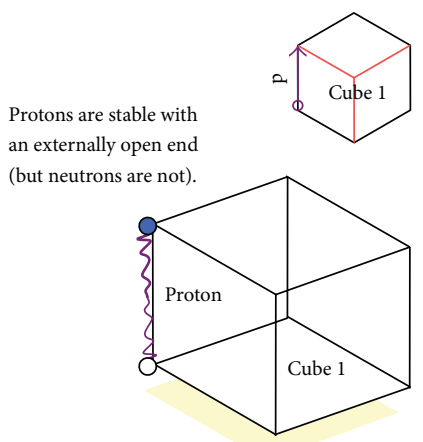

(a)

$$
{ }_{1} \mathrm{H}_{1} \begin{gathered}
\text { D, atomic hydrogen } \\
\text { (stable) (deuterium) }
\end{gathered}
$$

The stability of this nuclide is attributed to the single proton and neutron forming an overlapping linear structure using cisphasic bonds.
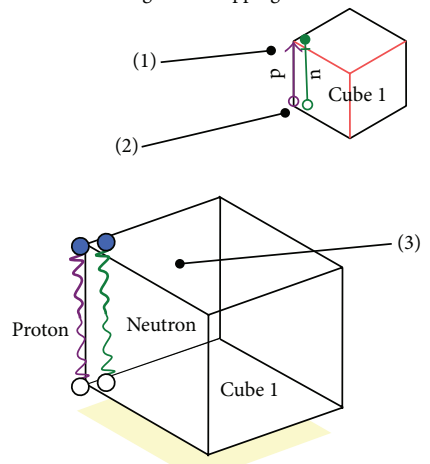

(b)

$$
\begin{array}{lr}
\hline \begin{array}{l}
\text { Relatively long half-life (12 yrs), } \\
\text { naturally occurring. }
\end{array} \mathrm{H}_{2} \begin{array}{c}
\text { (unstable) } \\
\text { (tritium) } \\
12.32 \mathrm{yr}
\end{array}
\end{array}
$$

Nuclides higher than ${ }_{1} \mathrm{H}_{1}$ require transphasic joints between neutrons, and these introduce instability. The longevity of ${ }_{1} \mathrm{H}_{2}$ is attributed to the simple unstrained assembly.

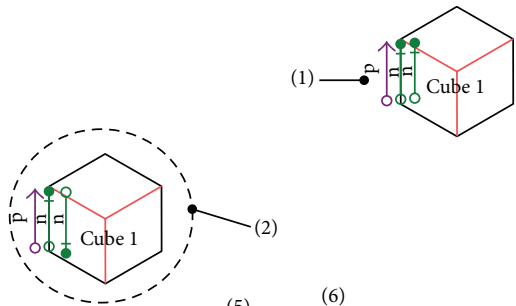

$$
{ }_{1} \mathrm{H}_{3} \begin{aligned}
& \text { (unstable) } \\
& 139 \mathrm{E}-24 \mathrm{~s}
\end{aligned}
$$

The additional neutron $\left(\mathrm{cf}_{1} \mathrm{H}_{2}\right)$ causes a major shape change.
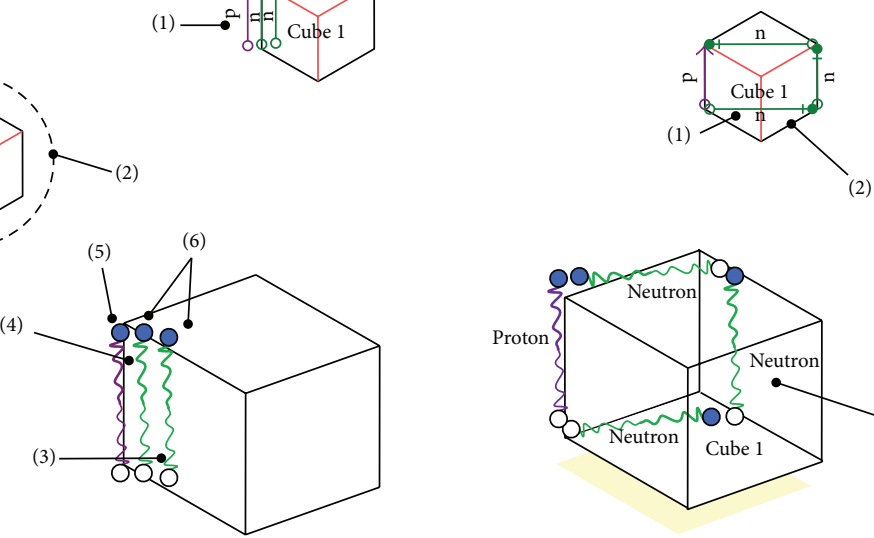

(c)

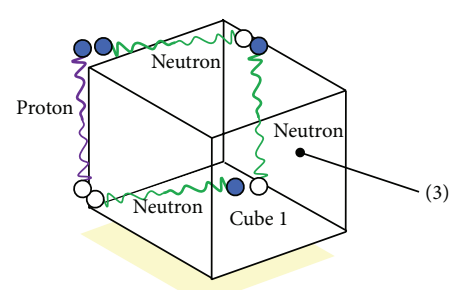

(d)

$$
{ }_{1} \mathrm{H}_{4} \stackrel{\text { (Unstable) }}{>910 \mathrm{E}-24 \mathrm{~s}}
$$

The stability is similar across this series, as these are all nonviable structures.

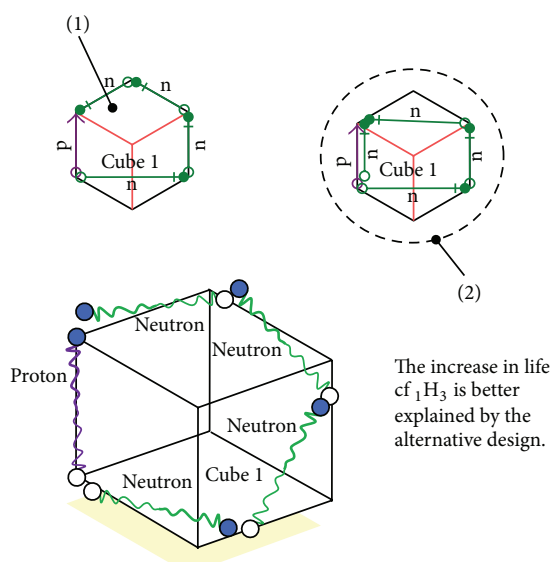

(e)

\section{${ }_{1} \mathrm{H}_{5} \begin{gathered}\text { (unstable) } \\ 290 \mathrm{E}-24 \mathrm{~s}\end{gathered}$}

The decreased life $\mathrm{cf}_{1} \mathrm{H}_{4}$ is attributed to the poor structure and the increased number of unstable neutrons involved.

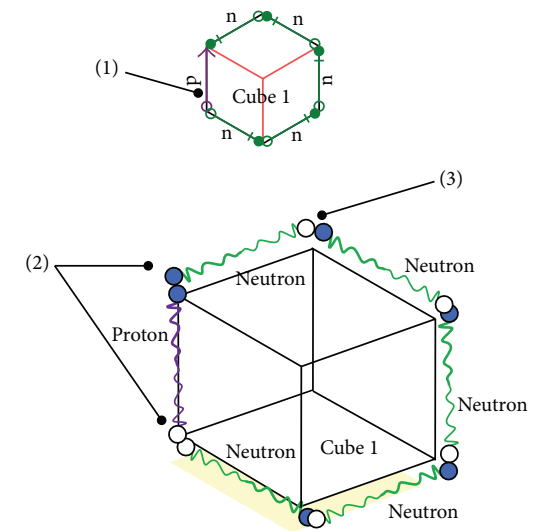

(f)

FIGURE 7: Continued. 


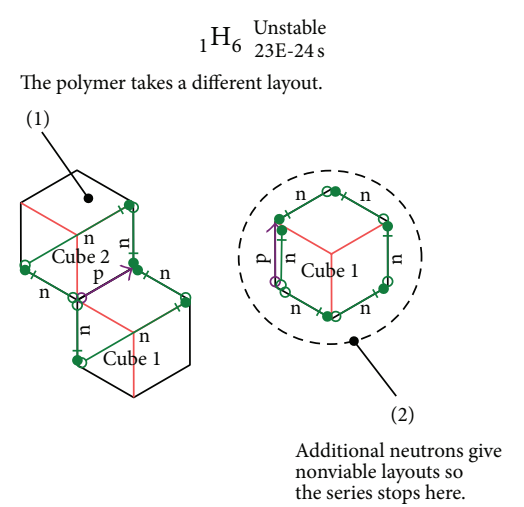

(g)

Figure 7: Internal structure of the nuclides of hydrogen, as proposed by the Cordus theory. In Figure (b) we have the following. (1) Simple pair: this comprises one proton and one neutron, making ${ }_{1} \mathrm{H}_{1}$. (2) All these are cisphasic joints and hence stability. (3) The neutron joins with the proton in a cisphasic relationship. Doing so gives stability advantages to the proton and neutron. The similar but nonidentical masses of the particules mean that there is a small degree of strain in the assembly. In Figure (c) we have the following. (1) This is the preferred design. (2) An alternative design. (3) Second neutron is also in a cisphasic relationship with the proton and first neutron. This is a type of bridge structure. The instability arises from the lack of orthogonality, that is, strain. (4) Primary neutron is in a cisphasic relationship with the proton. (5) proton. (6) neutron. In Figure (d) we have the following. (1) Lamellar plate, in condensed representation. (2) This subassembly is one of the permitted types. However, being the smallest of the subassemblies, the strain per joint is high. Hence, it has poor stability (except when balanced with 2 protons and 2 neutrons). (3) Lamellar plate. In Figure (e) we have the following. (1) Preferred design. (2) An alternative design. In Figure (f) we have the following. (1) The polymer wraps itself around the edges of a cube. (2) These are the only cisphasic joints in the assembly. (3) All the neutron-neutron joints are transphasic $(n \times n)$. in Figure $(g)$ we have the following. (1) No more neutrons can be fitted into a single cube, but a proton bridge structure is available. (2) An alternative design.

cubes. The network is primarily a closed loop of nucleons: there are no free reactive ends. The exceptions are certain light elements that have so few nucleons as not to be able to wrap completely around a cube, where open structures are permitted, terminated by protons [23]. However, for stability reasons, these may not be terminated by neutrons. Examples are provided below. These requirements arise by logical extension from the handed nature of the Cordus synchronous interaction (strong force), specifically the emission of discrete forces in three orthogonal emission directions.

With these concepts in place, the next idea in the Gedanken experiment is introduced: the morphology of the nuclear polymer and the nature of the bonds (cisand transphasic) are the primary determinants of stability/instability of the nuclide concerned [23]. Next, this principle is tested by application to two case studies: the hydrogen and helium nuclides.

4.9. Application to Simple Nuclei. With models in place for the cis- and transapplications of the strong force, there are sufficient basic concepts to identify the layout of the nuclear polymer for simple nuclides.

4.9.1. Hydrogen Nuclides. Hydrogen ${ }_{1} \mathrm{H}_{0}$ is simply the proton as already shown. It is proposed that the ${ }_{1} \mathrm{H}_{1}$ deuteron is a closed (parallel) cisphasic assembly of one proton and one neutron. The ${ }_{1} \mathrm{H}_{n}$ nuclides of hydrogen are expansions of the polymer by insertion of neutrons with transphasic bonds. The predicted nuclear polymers are shown in Figure 7. Note that in some cases there are alternative layouts. This is because the mechanics of the nuclear polymer theory allow more than one interpretation in certain cases.

This theory explains the stability trends in these nuclides.

(i) The stability of ${ }_{1} \mathrm{H}_{1}$ can be explained by the cisphasic proton-to-neutron bond.

(ii) Likewise, the long life of ${ }_{1} \mathrm{H}_{2}$ is attributed to its cisphasic bonds and its instability to the structure lacking orthogonality.

(iii) The poor viability of all the higher nuclides is explained by their transphasic neutron chains.

(iv) The theory also explains why the series stops where it does, at ${ }_{1} \mathrm{H}_{6}$. This is because there is a neat morphological boundary at ${ }_{1} \mathrm{H}_{6}$ such that the next longer polymers do not have access to a suitable layout.

4.9.2. Helium Nuclides. Explanations of the helium nuclides are given in Figure 8.

The helium nuclides are a strange series, because of abrupt changes and reversal in viability. This has historically been a difficult, even impossible, area for other theories. It is so problematic that some theories omit the light elements altogether. The Cordus theory has no such difficulties. It successfully explains the trends in stability. The explanation is entirely morphological and is based on the principle that certain combination of protons and neutrons cannot find a suitable shape and therefore cannot exist (except fleetingly). Hence, the difficult questions, which have troubled many other theories, can now be answered. 
${ }_{2} \mathrm{He}_{0}$ (does not exist)

There are no accessible layouts for two plain protons: none of the options shown here is viable. Hence, ${ }_{2} \mathrm{He}_{0}$ does not exist.
${ }_{2} \mathrm{He}_{1}$

This is the only stable assembly that breaks the rule of one neutron per proton. The proposed reason is that the structure is chirally complete despite exposed ends of the protons

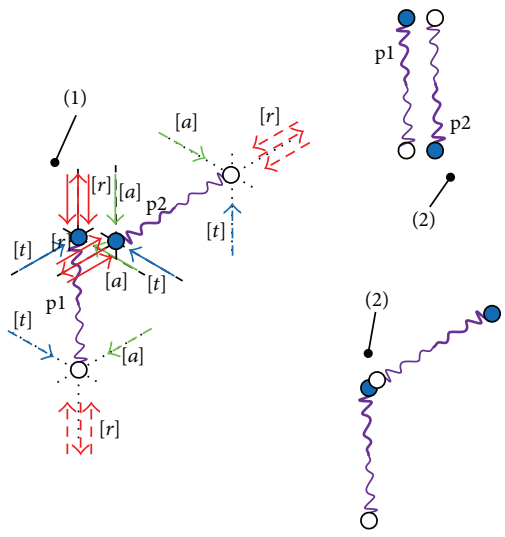

(a)

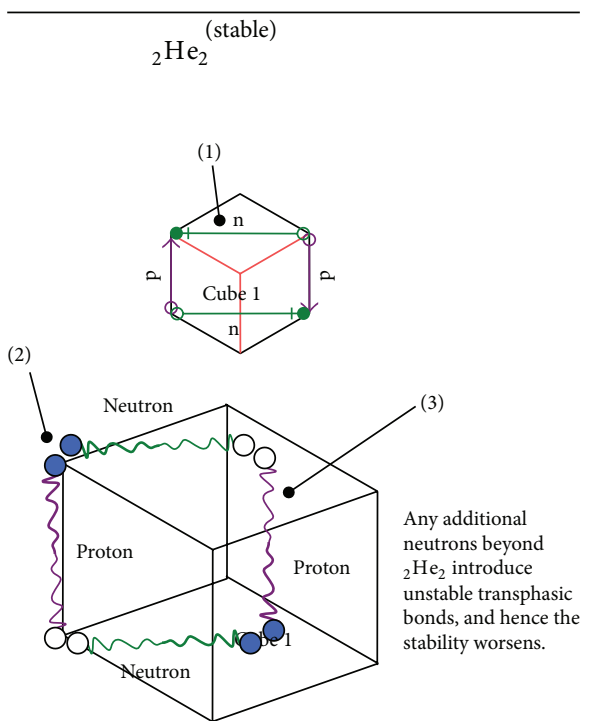

(c)

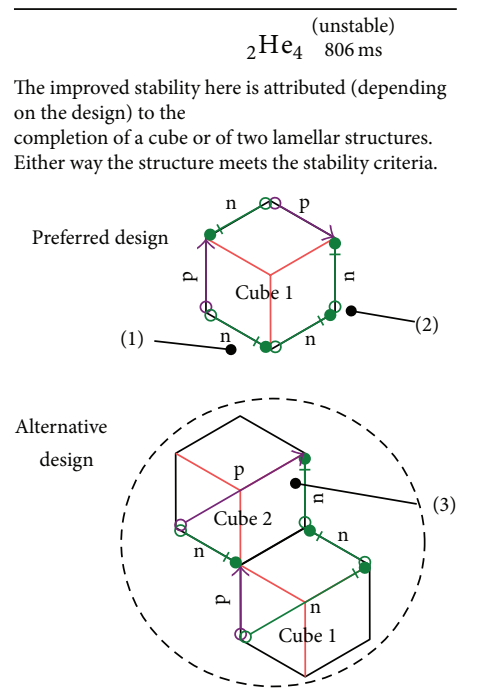

(e)
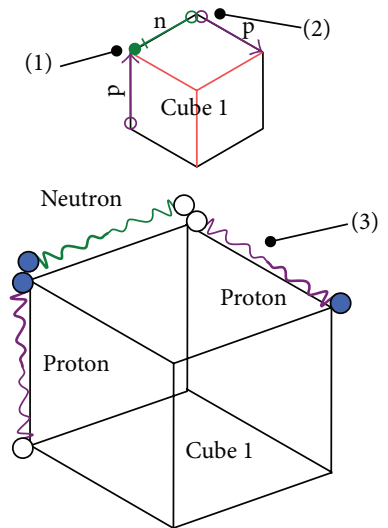

(b)

${ }_{2} \mathrm{He}_{3}<10 \mathrm{E}-15 \mathrm{~s}$

This nuclide struggles with viability due to the incomplete filling of the cube.

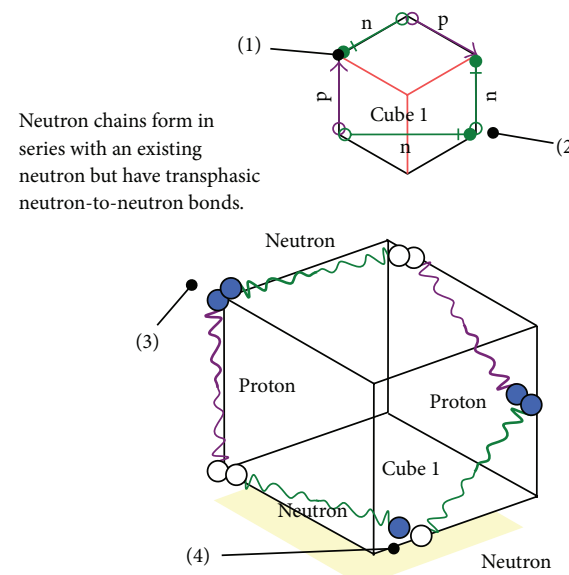

(d)

${ }_{2} \mathrm{He}_{5}$ (unstable)
There is no accessible symmetrical structure, and hence the nuclide is

There is no accessible symmetrical structure, and hence the nuclide is nonviable

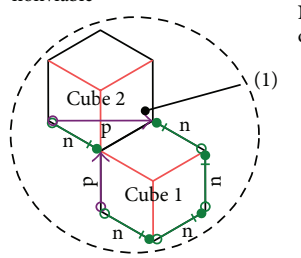

None of these

designs is viable
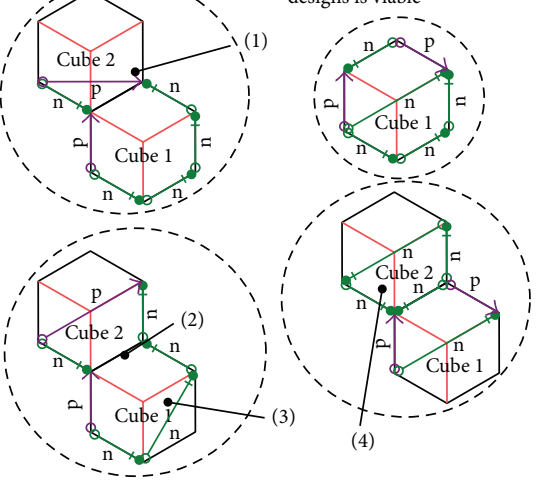

(f)

Figure 8: Continued. 


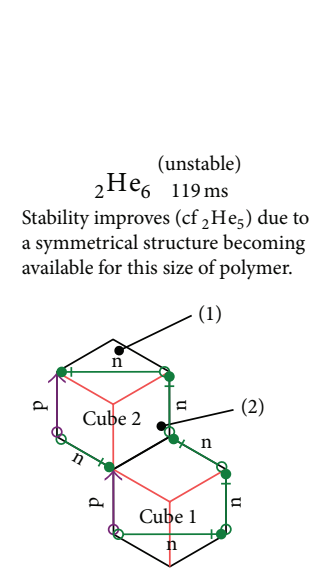

(g)

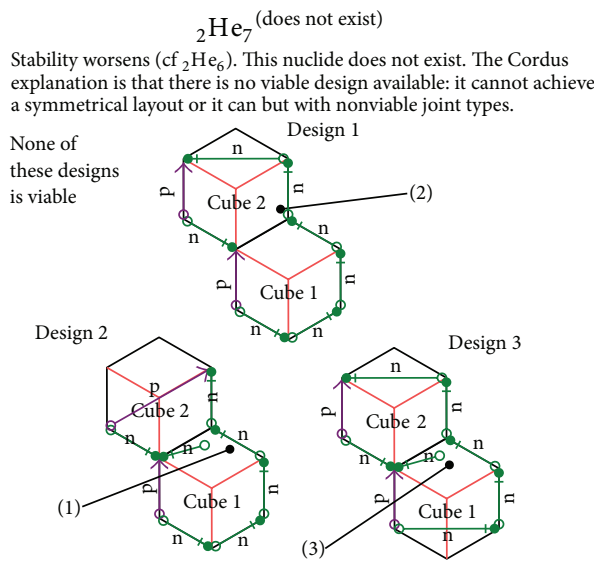

$(\mathrm{h})$

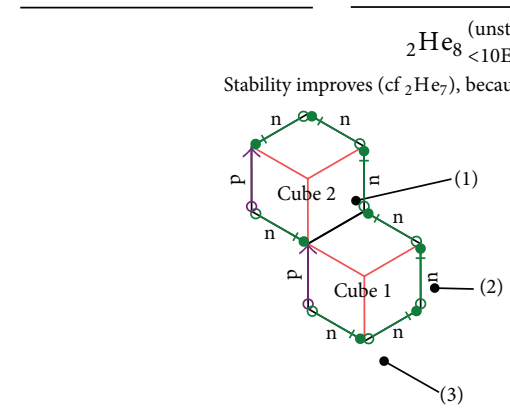

(i)

FIGURE 8: Internal structure of the nuclides of helium, as proposed by the Cordus theory. In Figure (a) we have the following. (1) Prohibited: two cisphasic protons do not give complete HEDs. (2) Cisphasic bonds are possible in principle but are nonviable without at least one neutron per cube. In Figure (b) we have the following: (1) Open polymer: protons are stable with an externally open end (but neutrons are not), which applies to ${ }_{2} \mathrm{He}_{1}$ (shown here) and ${ }_{1} \mathrm{H}_{0}$. (2) All these are cisphasic joints; this is proposed as the reason why the assembly is stable. (3) The two proton ends are open, because the structure is simple enough to be fully positionally determined. In Figure (c) we have the following. (1) Lamellar plate structure: four nucleons in a square, for ${ }_{2} \mathrm{He}_{2}$. This is the nominal representation: the actual shape expected to be equal to strain on all members; that is, the square is expected to be twisted. (2) Cisphasic joints throughout. These are stable. (3) Structure is likely bent out of plane too. In Figure (d) we have the following. (1) Incomplete cube is nonviable. (2) Additional neutrons assembled with transphasic bonds into an extended loop. The two protons are unaffected. (3) Strong cisphasic joints between proton and neutron. (4) Vulnerable transphasic joints between neutrons. In Figure (e) we have the following. (1) Additional neutron added to loop. (2) Cube is now full on all available sides. (3) Symmetrical lamellar structures. In Figure (f) we have the following. (1) Extremely poor filling of the cube makes for poor stability. (2) No bridge neutron possible. (3) Asymmetrical structure. (4) Lack of proton in this cube. In Figure (g) we have the following. (1) Structure comprises 2-linear incomplete symmetrical. (2) Balanced occupancy of cubes. In Figure (h) we have the following. (1) This assembly requires a bridge neutron, but there are insufficient protons to allow this. A proton is needed here to create a viable joint. (2) Lack of symmetry. (3) Not a viable joint. In Figure (i) we have the following. (1) Both cubes are full. (2) The long neutron chain results in poor viability. (3) The series stops here because an additional cube would require the availability of another proton.

(i) Why does not ${ }_{2} \mathrm{He}_{0}$ exist? Cisphasic bonds are possible in principle but are nonviable without at least one neutron per cube.

(ii) Why is ${ }_{2} \mathrm{He}_{1}$ stable, when the nuclides generally require $\mathrm{n} \geq \mathrm{p}$ ? Protons are stable with an externally open end (but neutrons are not).

(iii) Why are only ${ }_{2} \mathrm{He}_{1}$ and ${ }_{2} \mathrm{He}_{2}$ stable? Any additional neutrons beyond ${ }_{2} \mathrm{He}_{2}$ introduce unstable transphasic bonds, and hence the stability worsens.

(iv) Why do ${ }_{2} \mathrm{He}_{3}$ and ${ }_{2} \mathrm{He}_{5}$ have unexpectedly poor viability compared to the nuclides on each side? Their nuclear polymers are of a length that they do not have access to one of the viable cube-filling shapes. (v) Why are ${ }_{2} \mathrm{He}_{4}$ and ${ }_{2} \mathrm{He}_{6}$ viable whereas the nuclides on each side are not? The nucleons are sufficient to have access to viable shapes.

(vi) Why is the viability especially poor for ${ }_{2} \mathrm{He}_{7}$ ? There is no viable layout available.

(vii) Why does ${ }_{2} \mathrm{He}_{8}$ have such poor viability? The structure is sound, but the long neutron chain results in poor viability.

(viii) Why does the series stop at ${ }_{2} \mathrm{He}_{8}$ ? There are insufficient protons to expand into another cube structure. And a bridge neutron cannot be inserted into ${ }_{2} \mathrm{He}_{8}$ due to wrong end conditions. 
Some of these explanations require further details about the way the nuclear polymer fills the cubes, which is available elsewhere [23]. The shape progressions within the Cordus theory match the stability trends exactly. The theory is also successful at explaining why the series starts and stops where it does.

4.10. System Model. The concepts that have been presented here are a radical departure at the fundamental level and have profound implications for the directions taken by fundamental physics in the future. Almost all the ideas that have been presented in this paper are unorthodox, and conventional physics may need some time to evaluate their validity and digest the implications. To assist such an appraisal, a summary of the conceptual framework is shown in the system model of Figure 9. This diagram is represented in integration definition zero (IDEF0) system modeling notation [26]. There are five conceptual components to this theory, with relationships of causality between them. Those five components are models for the internal structure of the proton, neutron, the Cordus synchronous interaction and proposed cisphasic and transphasic bonding between nucleons. These are each described above, and the diagram summarizes their relationships.

\section{Discussion}

This paper provides new mechanics for the nuclides, one totally unlike any other theory. This provides a revolutionary new explanation of nuclear mechanics. The mechanics have been developed from first principles and then applied to the hydrogen and helium nuclides. They successfully explain why each nuclide is stable, unstable, or nonexistent. This is a breakthrough as this depth of explanation has not previously been achieved. The same mechanics also successfully explain the stability trends of all the nuclides up to and including $\mathrm{Ne}$ [23]. The explanations, while still at the qualitative stage of an early conceptual theory, are overwhelmingly better than anything possible from QCD theory and the nuclear models. The implications are profound, in those hidden-variable theories not quantum mechanics, which now appear to be the way forward for fundamental physics. These outcomes and their implications are elaborated below.

\subsection{What Has Been Achieved?}

\subsubsection{A Richer Understanding of the Synchronous Interaction} (Strong Force). The first contribution is the conceptual one of building a more nuanced concept for the strong force generally (Cordus: synchronous interaction). A radically different idea has been proposed for the strong interaction at the quark level and the strong nuclear force. Conventionally, the strong nuclear force overcomes the electrostatic repulsion of protons. In contrast, the Cordus theory proposes that the electromagnetic-gravitational (EMG) forces, for example, electrostatic forces between nucleons, are inoperative within a coherent body such as the nucleus (at least for small nuclei), and instead the synchronous force is manifest [17].
This is a radical departure from the orthodox perspective, which otherwise sees the strong and electrostatic forces as operating concurrently. The Cordus theory predicts that the interaction between neighboring protons in the nucleus is entirely synchronous (strong force) and that there is no electrostatic repulsion (at least for small nuclei). The corollary is that it predicts that the electrostatic force (and not the synchronous interaction) operates between free protons in discoherent assemblies. This may be falsifiable. A related contribution is the idea that the synchronous interaction makes two distinct types of bond, differentiated by same versus opposed phase (cis- and transphasic). This concept does not exist in conventional theories of the strong force.

5.1.2. Proton-Neutron Bonding and the Necessity of Neutrons. The second contribution is showing how the proton and neutron may be bonded. This has not previously been achieved. Existing theories like the shell and liquid drop models require but do not explain this, and so does QCD, which though it has a solution for the bonding of quarks via gluons, which does not explicitly explain the bonding between nucleons. The present work identifies an advantage to the proton in being bonded to a neutron, when otherwise there would seem no reason for such a bond. That advantage is the preferential alignment of discrete forces in three orthogonal directions. The neutrons are necessary because they provide a set of discrete forces that are complementary to those of the proton. The stable bonding within the nucleus occurs because of this synchronous compatibility and is proposed to have nothing to do with electrostatic charge per se. Thus, the Cordus theory predicts, in a major departure from conventional models of the nucleus, that the protons in stable nuclei are bound through neutrons, which are essential intermediaries, and that nuclear bonding involves synchronous interactions rather than charge per se. In contrast, other theories model the protons as being bound directly together or alternatively in an amorphous collection (liquid drop) or as shells. This difference may be testable and falsifiable. The Cordus theory predicts that proton-to-proton bonds may be either cisphasic or transphasic. This should be testable, given that the Cordus concept of frequency phase corresponds to the QM concept of spin, which in principle is measurable. The cisphasic bonds are predicted to be more stable than the transphasic.

5.1.3. Nuclear Polymer. A third contribution is the introduction of the concept of nuclear polymer including the provision of a logically consistent set of qualitative mechanics, the lemmas, governing its structure. The concept of chains and bridges of nucleons is novel and permits an entirely new approach to modeling nuclear structures. This provides a natural explanation for why the nucleus has physical size. Morphology is identified as the key determinant of nuclide stability, that is the way the polymer is draped over the available shapes. These shapes are also derivatives of the synchronous interaction. It is subsequently shown that this idea of nuclear polymers is powerful in its ability to explain other nuclides [23]. The Cordus theory predicts that the 


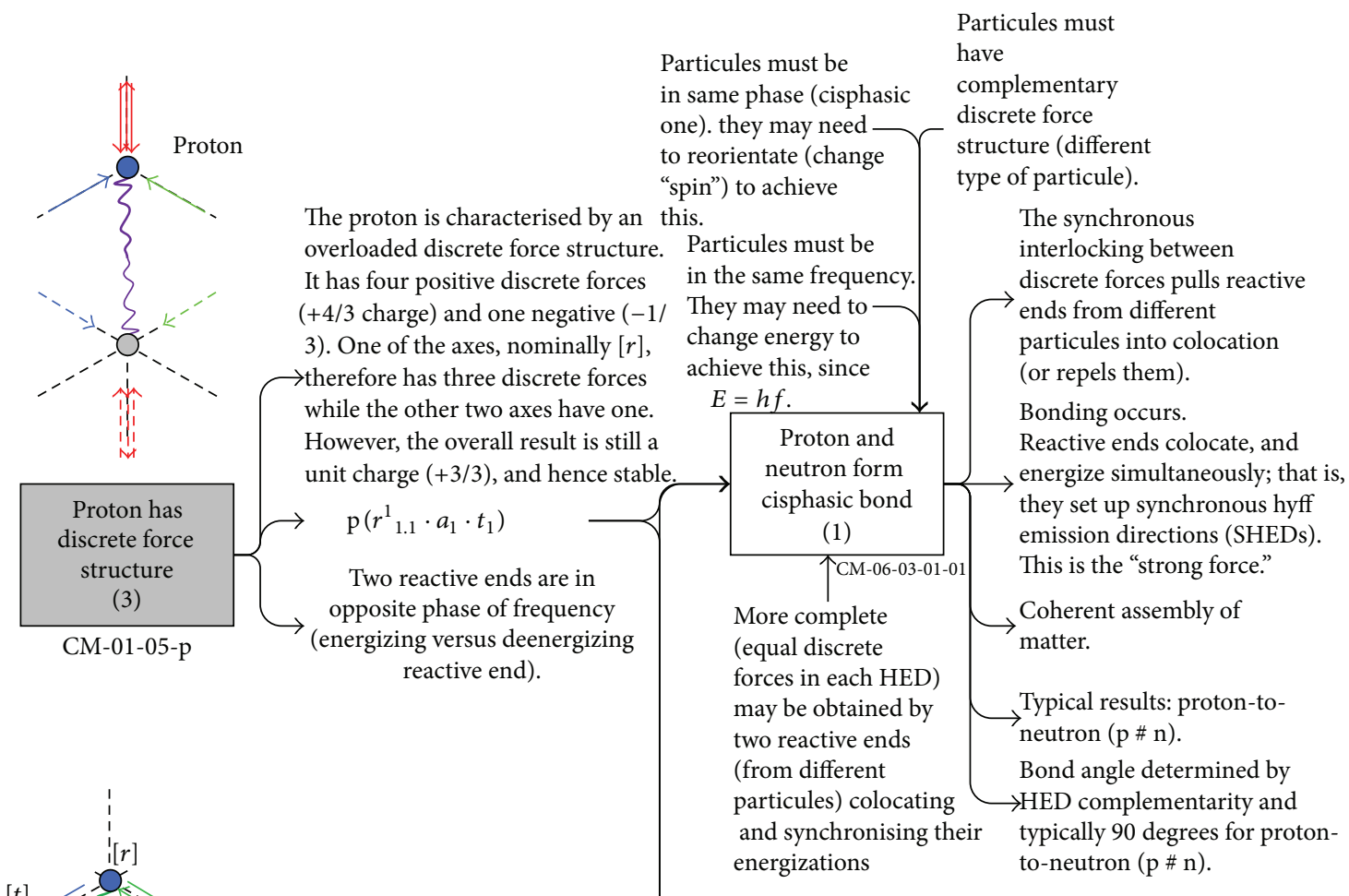

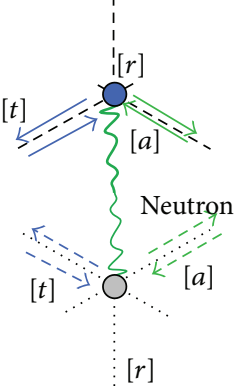

The neutron is characterised by having two discrete fields in each direction (charge) and is therefore neutral as a whole. However, it does not have full $\rightarrow$ HEDs in all three axes and is therefore intrinsically instable unless it is free to dynamically reallocate the discrete fields or

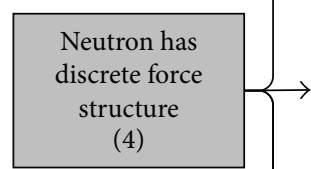

CM-01-05-n is bonded with a proton. $\mathrm{n}\left(r_{1}^{1} \cdot a \cdot t_{1}^{1}\right)$

Arrangement of discrete $\rightarrow$ fields is flexible in neutron: pairs may shift to $[a]$ axis.

Two reactive ends are in opposite phase of frequency (energizing versus deenergizing reactive end)

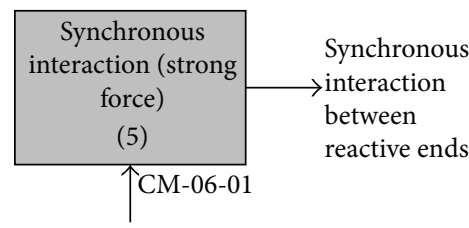

Interaction of discrete forces between particules, by negotiation of hyff emission directions (HEDs)
Particules must be ). they may need to reorientate (change "spin") to achieve

Particules must be change energy to chieve this, since

$\begin{gathered}\text { Proton and } \\ \text { neutron form } \\ \text { cisphasic bond } \\ (1)\end{gathered}$
TCM-06-03-01-01
(equal discrete
forces in each HED)
may be obtained by
two reactive ends
(from different
particules) colocating
and synchronising their
energizations

Particules must

complementary

tructure (different

The synchronous

interlocking between

orces pulls reactive

Reactive ends colocate, and

they set up synchronous hyff emission directions (SHEDs). the "strong force."

Coherent assembly of

Typical results: proton-toneutron ( $\mathrm{p} \# \mathrm{n}$ )

ED complem ypically 90 degrees for proton-

\section{个}

Particules must be in opposite phase Particules must (transphasic one). have the same They may need to discrete force reorientate (change structure (same "spin") to achieve type of particule).

this.

Particules must be in same frequency. They may need to change energy to achieve this, since $E=h f$.

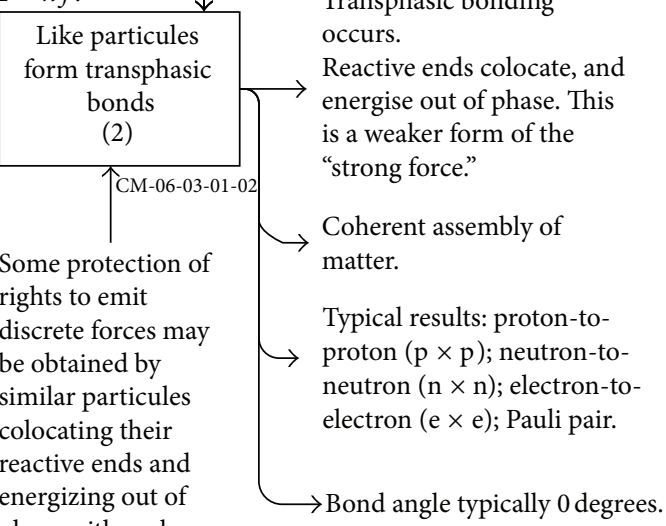
phase with each other.

FIGURE 9: System overview of the causes of bonding between protons and neutrons in the nucleus. The diagram summarizes the main features of the Cordus design of the proton: this is a nonlocal hidden-variable solution and it proposes that the proton has internal structures as well as a particular signature to the discrete forces (discrete fields) that it emits, likewise the neutron. The rest of the diagram identifies the two different types of synchronous (strong) bond being proposed here, namely, the cisphasic and transphasic states. 
nucleus is best described as a nuclear polymer of proton-toneutron joints with cisphasic bonds. Coupled with the Cordus concept that these particules have finite span (distance between reactive ends), this further predicts that the nucleus has physical size. The Cordus theory specifically rejects the zero-dimensional point construct of QM. This prediction of physical size is consistent with the empirical observation that nuclei have size (measured as charge radius). By comparison, quantum theory cannot explain how aggregates of point particles should have physical size.

5.1.4. Models of Simple Nuclei. A fourth contribution is the provision of candidate descriptive physical models for the proton, neutron, deuteron, and several simple nuclide assemblies thereof. The Cordus theory predicts that the proton and neutron have specific internal structures and specific signatures for the emission of discrete forces. This may be falsifiable. An associated contribution is the prediction of the internal structure of the hydrogen and helium nuclides and an explanation of their stability trends. The Cordus theory explains qualitatively why the stability worsens with increased neutron count. It also explains the discontinuities in stability, for example, why ${ }_{2} \mathrm{He}_{5}$ is so much less stable than its neighboring isotopes. The limits of the series, why they stop where they do, are also explained. Another important explanation is why ${ }_{2} \mathrm{He}_{1}$ should be stable, as the only stable nuclide with $\mathrm{p}>\mathrm{n}$. No other theory can explain all these effects, and most of the theories, for example, liquid drop and QCD, cannot explain any of these. So the contribution is an improved explanation for the $\mathrm{H}$ and $\mathrm{He}$ nuclides.

A final contribution is methodological, in that the work demonstrates (a) the vitality and relevance of nonlocal hidden-variable theories, which otherwise have been rejected without proper consideration, and (b) the value in using a system design approach for concept development. The resulting Cordus theory provides new insights and fresh ideas towards an old problem. This has otherwise not been achieved with other hidden-variable solutions, for example, de Broglie-Bohm.

The explanations of the Cordus theory are consistent with empirical evidence of how matter behaves under the strong force, so the theory has construct validity. The theory also has good external validity in that it readily generalizes to a wide range of different phenomena in physics, even cosmological $[19,20]$. By comparison, other nuclear models only apply to a limited set of situations.

5.2. Implications. The points of difference of the Cordus theory arise from several conceptual attributes that are radically different to any other theory of physics including QM and QCD.

The first is the proposition that the nucleons have geometric span. Hence, the Cordus theory rejects the zerodimensional point construct of QM. It is the $0 \mathrm{D}$ point thinking and the resulting singularities that frame the orthodox paradigm into the excessively limiting idea that the nuclear bonding force is repulsive at short range, strong at middle range, and weak at long range. This odd set of properties is a consequence of the premise and does not need to be a physical reality. The Cordus theory proposes that it is better to consider the nuclear bonding force as a synchronous interaction. The Cordus particule idea provides physical interpretations for superposition, entanglement, spin, frequency, phase, orientation angles (e.g., polarity), and parity violation which are otherwise indefinable in QM. Thus, quantum mechanics (QM) is reinterpreted as a solution of averages, applicable only to coherent bodies where the size of the particles can be neglected and otherwise not applicable at both the subatomic and macroscopic scales.

The second is that the two ends of a particule energize and deenergize in sequence, at a frequency. Consequently the whole particule is not in a single state. This is consistent with the QM concept of geometric superposition. In QM, this is attributed to a fundamental stochastic variability, but the Cordus theory rejects QM's interpretation as a simplistic and coarse approximation to a deeper deterministic causality. Furthermore, the Cordus theory outright rejects the QM idea that a particle can be in a temporal superposition.

The third unique feature of the Cordus theory is its concept for the strong force. This is reformulated as a synchronous interaction between discrete forces [23]. This is in contrast to the QCD concept of an exchange of bosons. This has far-reaching and profound implications for nuclear bonding and cosmology, explored in other papers.

Unique to this theory is the concept that bonds between nucleons may take two forms: cis- and transphasic [23]. This feature arises from the nature of the proposed synchronous interaction. It is in stark contrast to the single interaction envisaged for bonding by all other theories. There is nothing like this in any of the other nuclear theories nor in QCD. Furthermore, the Cordus theory predicts that nucleons are arranged geometrically in space, according to specific rules of orientation, and hence the nuclear polymer idea.

Consequently this theory predicts that there are several ways, not only one as in the orthodox theories, for how protons and neutrons may bond. Having multiple interactions also provides a means to differentiate between stable and unstable bonds in nuclides. The principles presented here, specifically the cis- and transphasic bonds, have the potential to support the development of a profoundly different theory of nuclear structure. It is expected that at a deeper level there may be some similarities with the liquid drop model and SEMF. This is because all of these are models that consider the "geometric packing of the nucleons" [23]. However, the point of difference is that the Cordus theory offers a solution for internal mechanics and structure of the nucleus and how the individual protons and neutrons are bound together in space. In contrast, the other theories merely consider the nucleons as an aggregated collection (hence drop or bag descriptions) and are unable to say anything about the internal distribution of the nucleons or their specific relationships to each other. In this theory, the nucleus structure is not a simple assembly of points nor a packing of spheres, but an assembly of rod-like structures into three-dimensional chains of protons and neutrons. 
5.3. Limitations. The Cordus theory is currently mostly a conceptual work. It does not calculate the binding forces, lifetimes, or the charge radii. So it is not a complete description of every feature of the strong force. This is a limitation but hardly a fatal one as neither does any other theory give quantitative predictions from first principles for all these variables (except by parameter-fitting and tuning).

There are two other features which might seem limitations of this theory, but they are not. The fact that this is a nonlocal hidden-variable design is not a limitation. It is only local hidden-variable solutions that are precluded by the Belltype inequalities $[2,3]$. The lack of a mathematical formalism is also not a limitation. Instead, it is merely a consequence of using the Gedankenexperiment method for conceptual development. This is a well-established method for concept development. The nature of the work, which is conceptual, does not at this stage in its development lend itself to a mathematical formalism. Mathematical development can follow as a future opportunity. The real limitation is one caused by the design method. Design converges on a sufficient solution, and therefore one cannot be certain that this is the only or the best solution. Thus, the design method, while good at fitting form to function, could result in spurious attribution of causal relationships. Nonetheless, this theory is not alone as other methodologies, most especially mathematical and statistical modeling, have the same limitation. The real tests are whether the theory put forward here advances the understanding of the nucleus, is internally self-consistent, and has predictive power. The evidence is that it does all those things.

5.4. Future Research. The Cordus nuclear theory has potentially far-reaching and deep implications for the future direction of fundamental physics. It demonstrates that a hidden-variable design has better explanatory power than other theories and models based on the $0 \mathrm{D}$ point premise. Given this and that it subsumes quantum mechanics, the implication is that the next deeper level of physics is better described by hidden-variable designs than quantum mechanics. The consequences are wide-ranging, in that a large number of physical phenomena can be described with the Cordus mechanics. The implications for future research are therefore vast. The theory has the potential to touch every area of fundamental physics and transform the conceptual foundations on which many theories are built, thereby requiring reformulation of those theories to accommodate covert structures.

Regarding the nuclides specifically, opportunities for further work lie in several directions. One is to develop a mathematical formulism for the synchronous interaction (including its directional, discrete, and phasic attributes), quantify the parameters, and evaluate the robustness of the resulting model against empirical data. There is also a need to develop a mathematical representation of the nuclear polymer and the filling of cubic structures. This would be helpful in exploring the heavier nuclides. Another opportunity is to further develop the concept and see whether the theory can explain additional nuclides. There is a need, at present unfulfilled by quantum theory, QCD, nuclear models, or string/M theory, to explain why any given nuclide is stable or unstable. There are also a large number of deviations in the trends that need explaining. Why are more neutrons required for stability in heavier nuclides; that is, why does the trend deviate from $\mathrm{p}=\mathrm{n}$ ? Why are some nuclides (e.g., ${ }_{4} \mathrm{Be}_{4}$ ) unexpectedly unstable? Why are the margins of nonexistence (drip lines) jagged? Why do some elements have only one stable nuclide, whereas others have multiple ones? No existing theory can do any of this, so it is a formidable challenge. There are many other nuclides to explain, with odd trends apparent even within one isotope series and hence much opportunity for future work and theory-building. Another opportunity for research is to model binding energy and charge radii. A complete theory of the nucleus will also need to explain the decay process itself and the weak interactions (beta plus and minus decay and electron capture), and the Cordus theory has already made progress in this area [25].

Being able to sketch out a coherent causality starting from the fundamental level, via the nucleus, and onwards to qualitative explanations for stability of the nuclides is a significant accomplishment. It gives reason to be optimistic that another breakthrough may be achievable, the creation of a consistent theory that spans fundamental physics and chemistry. Chemical interactions are primarily mediated by the electrons between interacting atoms. Consequently, future work could be focused on integrating the electrons into the nuclear polymer theory and explaining electron valance.

\section{Conclusions}

The purpose of this paper was to develop a systematic theory to explain the relationships between nucleons and thereby explain the stability, instability, and nonexistence states of the nuclides. This has been achieved in a Gedankenexperiment. The work reconceptualizes the basic principles of the bonding of protons and neutrons, using inferences from the Cordus theory for the synchronous interaction (strong force). The resulting theory predicts that protons and neutrons may form different types of bonds, with different stability. Specifically, the synchronous interaction assembles particules in- and out-of-phase (cis- and transphasic resp.) and into open or closed chains. The theory identifies the role of the neutrons in nuclear bonding. The protons in stable nuclei are bound through neutrons, which are essential intermediaries. Nuclear bonding therefore involves synchronous interactions rather than charge per se. In contrast, other theories model the protons as being bound directly together or alternatively in an amorphous collection (liquid drop) or as shells. The theory predicts that the assembly structures involve a nuclear polymer, and the mechanics of this have been anticipated. The concept of chains and bridges of nucleons is novel and permits an entirely new approach to theorizing nuclear structures. This is shown to readily provide a natural explanation for why the nucleus has physical size. Furthermore, morphology is identified as the key determinant of nuclide stability, that is the way the polymer is draped over the available shapes. These shapes are also derivatives of the synchronous interaction. Application of the theory to the hydrogen and helium nuclides shows that it successfully explains the trends in stability. This is important as it demonstrates, for the 
first time, a theory for nuclear structure starting from the strong/synchronous force at the fundamental level.

This theory opens up a new field of mechanics, offers a different conceptual framework for the nucleus, and advances the understanding of nuclear physics. It is a different concept to other theories and exceeds them in ability to explain stability trends for the nuclides. That it has been possible to achieve this from the hidden-variable sector, when no quantum theory has answered these questions, shows that serious consideration must now be given to the likelihood that particules may have internal structure after all. If so, the theory has the potential to revolutionize fundamental physics of the nucleus.

\section{Conflict of Interests}

The authors declare that there is no conflict of interests regarding the publication of this paper. Neither was the research conducted with personal financial benefit from any third party funding body, nor did any such body influence the execution of the work.

\section{Authors' Contribution}

Dirk J. Pons, Arion D. Pons, and Aiden J. Pons developed the conceptual foundation and the theory and critically evaluated the logical consistency thereof. All authors contributed to the writing of the paper. Dirk J. Pons created the drawings.

\section{References}

[1] A. Einstein, B. Podolsky, and N. Rosen, "Can quantummechanical description of physical reality be considered complete?” Physical Review, vol. 47, no. 10, article 777, 1935.

[2] J. S. Bell, "On the Einstein podolsky rosen paradox," Physics, vol. 1, no. 3, pp. 195-200, 1964.

[3] A. J. Leggett, "Nonlocal hidden-variable theories and quantum mechanics: an incompatibility theorem," Foundations of Physics, vol. 33, no. 10, pp. 1469-1493, 2003.

[4] S. Gröblacher, T. Paterek, R. Kaltenbaek et al., "An experimental test of non-local realism," Nature, vol. 446, no. 7138, pp. 871-875, 2007.

[5] L. de Broglie, "Recherches sur la théorie des quanta (researches on the quantum theory)," Annales de Physique, vol. 3, no. 10, 1925, http://tel.archives-ouvertes.fr/docs/00/04/70/78/PDF/tel00006807.pdf.

[6] D. Bohm and J. Bub, "A proposed solution of the measurement problem in quantum mechanics by a hidden variable theory," Reviews of Modern Physics, vol. 38, pp. 453-469, 1966.

[7] D. J. Pons, A. D. Pons, A. M. Pons, and A. J. Pons, "Wave-particle duality: a conceptual solution from the cordus conjecture," Physics Essays, vol. 25, no. 1, pp. 132-140, 2012.

[8] J. P. Elliott, "The interacting boson model of nuclear structure," Reports on Progress in Physics, vol. 48, no. 2, pp. 171-221, 1985.

[9] M. Gell-Mann, "Symmetries of baryons and mesons," Physical Review, vol. 125, no. 3, pp. 1067-1084, 1962.

[10] Y. Nambu and M. Y. Han, "Three triplets, paraquarks, and 'colored' quarks," Physical Review D, vol. 10, no. 2, pp. 674-683, 1974.
[11] G. Gamow, "Mass defect curve and nuclear constitution," Proceedings of the Royal Society of London, Series A, vol. 126, no. 803, pp. 632-644, 1930.

[12] C. F. V. Weizsäcker, “The theory of nuclear mass," Zeitschrift für Physik, vol. 96, no. 7-8, pp. 431-458, 1935.

[13] D. Ivanenko, "Remarks on the Establishing the Theory of Nuclear Forces," Progress of Theoretical Physics Supplement, vol. 85, pp. 20-26, 1985.

[14] T. Otsuka, A. Arima, and F. Iachello, "Nuclear shell model and interacting bosons," Nuclear Physics A, vol. 309, no. 1-2, pp. 1-33, 1978.

[15] W. Pfeifer, An Introduction to the Interacting Boson Model of the Atomic Nucleus, Vdf-Hochschulverlag AG an der ETH Zürich, 1998, http://www.uni-kassel.de/upress/online/frei/3-7281-25202.volltext.frei.pdf.

[16] I. Angeli, "A consistent set of nuclear rms charge radii: properties of the radius surface $R(N, Z)$," Atomic Data and Nuclear Data Tables, vol. 87, no. 2, pp. 185-193, 2004.

[17] D. Pons, A. Pons, and A. Pons, "Synchronous interlocking of discrete forces: strong force reconceptualised in a NLHV solution," Applied Physics Research, vol. 5, no. 5, 2013.

[18] D. J. Pons, A. D. Pons, and A. J. Pons, "Differentiation of matter and antimatter by hand: internal and external structures of the electron and antielectron," Physics Essays, vol. 27, no. 1, pp. 2635, 2014.

[19] D. J. Pons, A. D. Pons, and A. J. Pons, "Time: an emergent property of matter," Applied Physics Research, vol. 5, no. 6, pp. 23-47, 2013.

[20] D. J. Pons, "Outer boundary of the expanding cosmos: discrete fields and implications for the holographic principle," The Open Astronomy Journal, vol. 6, no. 1, pp. 77-89, 2013.

[21] F. Laudisa, "Non-local realistic theories and the scope of the Bell theorem," Foundations of Physics, vol. 38, no. 12, pp. 1110-1132, 2008.

[22] F. de Zela, "A non-local hidden-variable model that violates Leggett-type inequalities," Journal of Physics A: Mathematical and Theoretical, vol. 41, no. 50, Article ID 505301, 2008.

[23] D. J. Pons, A. D. Pons, and A. J. Pons, "Explanation of the table of nuclides: qualitative nuclear mechanics from a NLHV design," Applied Physics Research, vol. 5, no. 6, pp. 145-174, 2013.

[24] D. J. Pons, A. D. Pons, and A. J. Pons, "Annihilation mechanisms," Applied Physics Research, vol. 6, no. 2, 2014.

[25] D. J. Pons, A. J. Pons, and A. J. Pons, "Beta decays and the inner structures of the neutrino in a NLHV design," Applied Physics Research, vol. 6, no. 3, pp. 50-63, 2014.

[26] FIPS, "Integration Definition for Function Modeling (IDEF0)," 1993, http://www.itl.nist.gov/fipspubs/idef02.doc. 

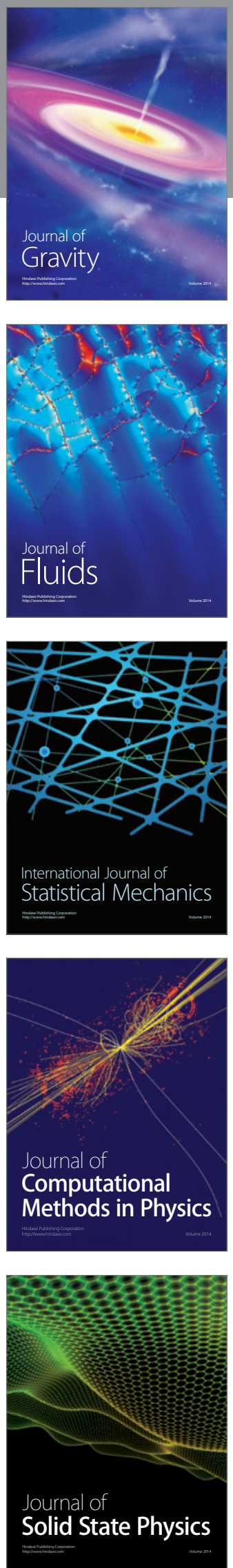

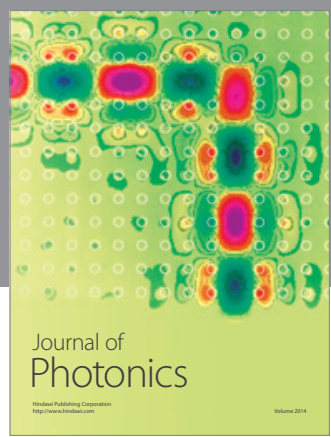

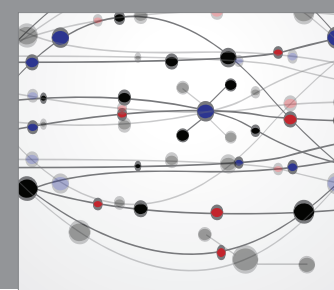

The Scientific World Journal

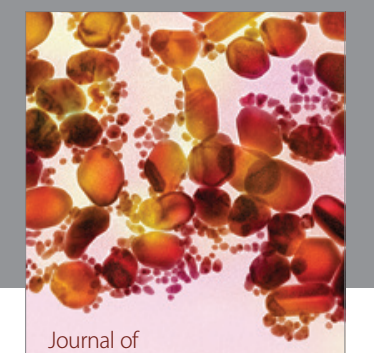

Soft Matter
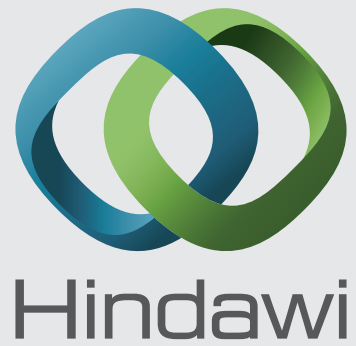

Submit your manuscripts at

http://www.hindawi.com
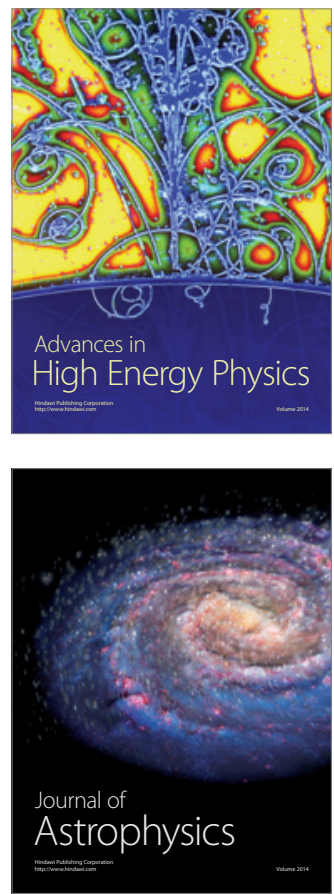
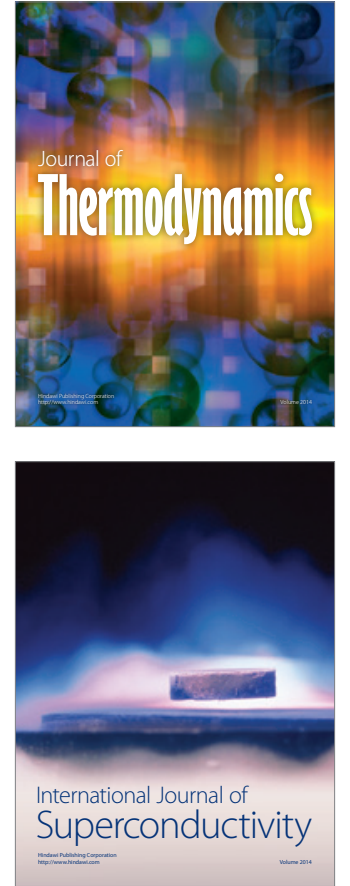
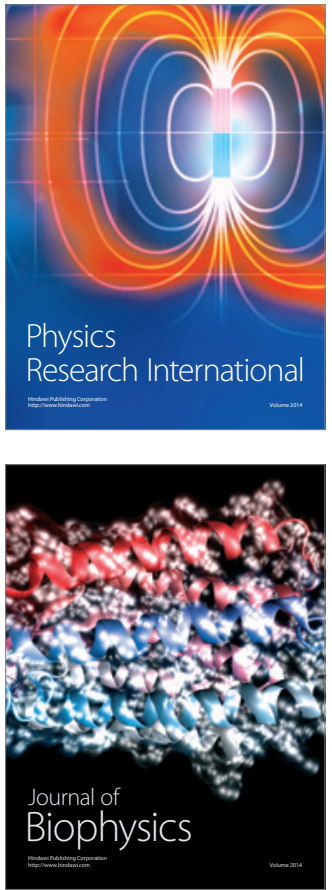
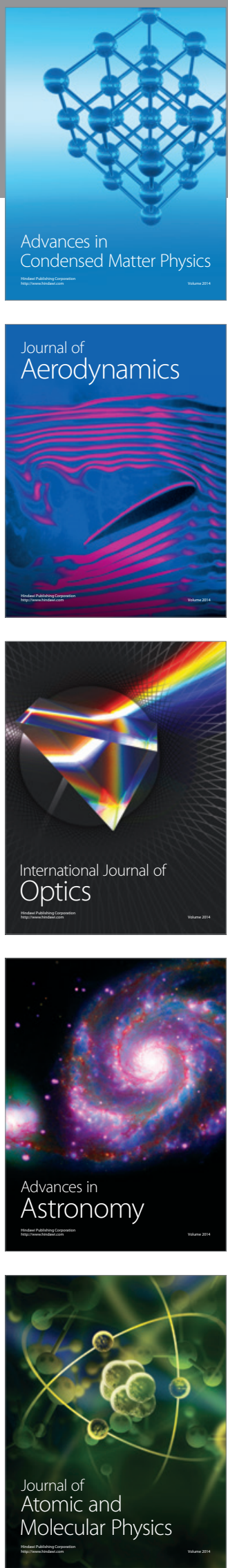